Proceedings of the 2017 CERN-Latin-American School of High-Energy Physics, San Juan del Rio, Mexico 8-21 March 2017, edited by M. Mulders and G. Zanderighi, CYR: School Proceedings, Vol. 4/2018, CERN-2018-007-SP (CERN, Geneva, 2018)

\title{
QCD AND THE PHYSICS OF HADRONIC COLLISIONS
}

\author{
M. L. Mangano
}

Theoretical Physics Department, CERN, Geneva, Switzerland

\begin{abstract}
This series of lectures reviews the basic principles underlying the use of quantum chromodynamics in understanding the structure of high- $Q^{2}$ processes in high-energy hadronic collisions. Several applications of relevance to the LHC are illustrated.
\end{abstract}

Keywords

Lectures; QCD; hadronic collisions; gluon emission; hadronization; jets

\section{Introduction}

The initial state of any LHC collision is formed by a pair of protons. Whether the hard process we are interested in is of electroweak origin (e.g. $W$ production) or a strong-interaction process (e.g. the production of jets, or the production of a pair of gluinos in Supersymmetry), its description requires the understanding of the structure of the proton. Quantum Chromodynamics (QCD) is the theory that describes the structure of the proton, and is therefore the starting point of any study of LHC physics. QCD is formulated in terms of elementary fields (quarks and gluons), whose interactions obey the principles of a relativistic quantum field theory, with a non-Abelian gauge invariance SU(3). To review the emergence of QCD as a theory of strong interactions, analysing the various experimental data and the theoretical ideas available in the years 1960-1973 (see, for example, Refs. [1,2]), would require more time than I have available. I shall therefore assume that you all know more or less what QCD is: that hadrons are made of quarks, that quarks are spin-1/2, colour-triplet fermions, interacting via the exchange of an octet of spin-1 gluons; I assume you know the concept of running couplings, asymptotic freedom and of confinement. I shall finally assume that you have some familiarity with the fundamental ideas and formalism of quantum field theory (Feynman rules, renormalization, gauge invariance), even though I shall make only very limited use of them.

In these lectures I shall focus on some elementary applications of QCD in high-energy phenomena. The material covered in these lectures includes the following:

1. The structure of the proton

2. The evolution of final states: from quarks and gluons to hadrons

3. Some key hard processes in hadron-hadron collisions: formalism, $W / Z$ production, jet production

The treatment will be very elementary, and the emphasis will be on basic and intuitive physics concepts. Given the large number of papers that contributed to the development of the field, it is impossible to provide a complete and fair bibliography. I therefore limit my bibliography to some excellent review books, and to references to some of the key results discussed here. For an excellent description of the early ideas about quarks, the classic reference is Feynman's book [3]. For a general, but rather formal, introduction to QCD, see, for example, Ref. [4]. For a more modern and pedagogical introduction, in the context of introductory course to field theory, use the excellent book by Peskin [5]. For a general introduction to collider physics, see Ref. [6]. For QCD applications to LEP, Tevatron and LHC, see Ref. [7] and, specifically for the LHC, see Ref. [8]. Explicit calculations, including the nitty-gritty details of next-to-leading-order (NLO) calculations and renormalization, are given in great detail for several concrete cases of interest in Ref. [12]. Many of the ideas used in my lectures are inspired by the very physical perspective presented in Ref. [13]. Papers on specific items can easily be found by consulting the standard hep-th and hep-ph preprint archives. 


\section{QCD and the proton structure at large $Q^{2}$}

The understanding of the structure of the proton at short distances is one of the key ingredients to be able to predict cross-sections for processes involving hadrons in the initial state. All processes in hadronic collisions, even those intrinsically of electroweak nature such as the production of $W / Z$ bosons or photons, are in fact induced by the quarks and gluons contained inside the hadron. In this lecture I shall introduce some important concepts, such as the notion of partonic densities of the proton, and of parton evolution. These are the essential tools used by theorists to predict production rates for hadronic reactions.

We shall limit ourselves to processes where a proton-(anti)proton pair collides at large centre-ofmass energy $(\sqrt{S}$, typically larger than several hundred $\mathrm{GeV})$ and undergoes a very inelastic interaction, with momentum transfers between the participants in excess of several GeV. The outcome of this hard interaction could be the simple scattering at large angle of some of the hadron's elementary constituents, their annihilation into new massive resonances, or a combination of the two. In all cases the final state consists of a large multiplicity of particles, associated to the evolution of the fragments of the initial hadrons, as well as of the new states produced. As discussed below, the fundamental physical concept that makes the theoretical description of these phenomena possible is 'factorization', namely the ability to isolate separate independent phases of the overall collision. These phases are dominated by different dynamics, and the most appropriate techniques can be applied to describe each of them separately. In particular, factorization allows one to decouple the complexity of the proton structure and of the finalstate hadron formation from the elementary nature of the perturbative hard interaction among the partonic constituents.

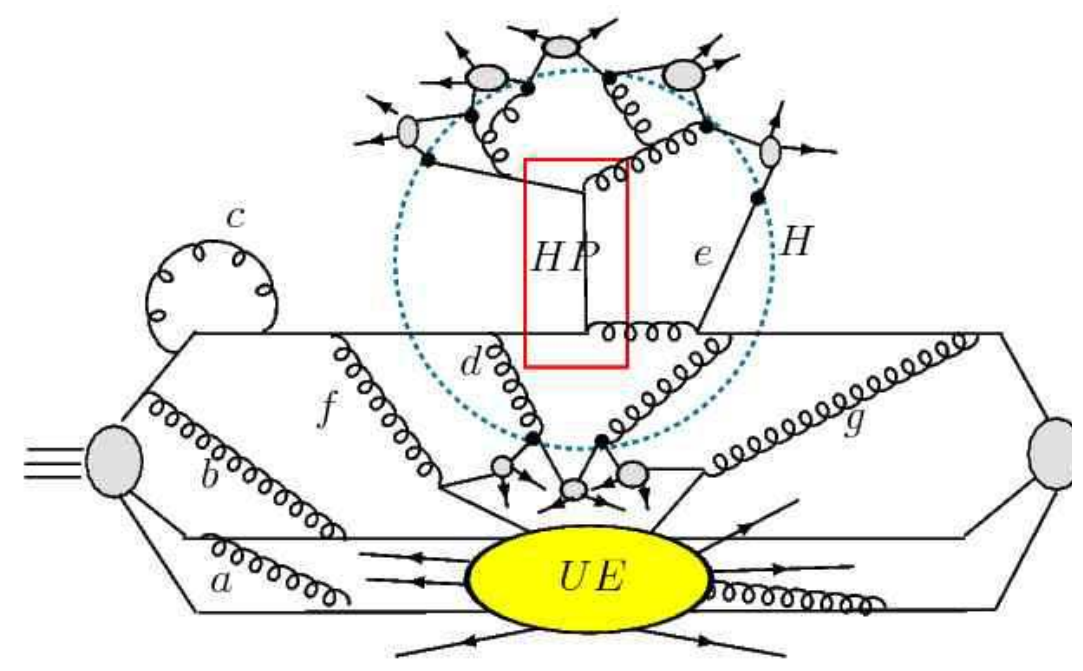

Fig. 1: General structure of a hard proton-proton collision

Figure 1 illustrates how this works. As the left proton travels freely before coming into contact with the hadron coming in from the right, its constituent quarks are held together by the constant exchange of virtual gluons (e.g. gluons $a$ and $b$ in the picture). These gluons are mostly soft, because any hard exchange would cause the constituent quarks to fly apart, and a second hard exchange would be necessary to reestablish the balance of momentum and keep the proton together. Gluons of high virtuality (gluon $c$ in the picture) prefer therefore to be reabsorbed by the same quark, within a time inversely proportional to their virtuality, as prescribed by the uncertainty principle. The state of the quark is, however, left unchanged by this process. Altogether this suggests that the global state of the proton, although defined by a complex set of gluon exchanges between quarks, is nevertheless determined by interactions which have a time scale of the order of $1 / m_{p}$. When seen in the laboratory frame where the proton is 
moving with energy $\sqrt{S} / 2$, this time is furthermore Lorentz dilated by a factor $\gamma=\sqrt{S} / 2 m_{p}$. If we disturb a quark with a probe of virtuality $Q \gg m_{p}$, the time frame for this interaction is so short $(1 / Q)$ that the interactions of the quark with the rest of the proton can be neglected. The struck quark cannot negotiate with its partners a coherent response to the external perturbation: it simply does not have the time to communicate to them that it is being kicked away. On this time scale, only gluons with energy of the order of $Q$ can be emitted, something which, to happen coherently over the whole proton, is suppressed by powers of $m_{p} / Q$ (this suppression characterizes the 'elastic form factor' of the proton). In this figure, the hard process is represented by the rectangle labelled HP. In this example a head-on collision with a gluon from the opposite hadron, leads to a $q g \rightarrow q g$ scattering with a momentum exchange of the order of $Q$. This and other possible processes can be calculated from first principles in perturbative QCD.

When the constituent is suddenly deflected, the partons that it had recently radiated cannot be reabsorbed (as happened to gluon $c$ earlier) because the constituent is no longer there waiting for the partons to come back. This is the case, for example, of the gluon $d$ emitted by the quark, and of the quark $e$ from the opposite hadron; the emitted gluon got engaged in the hard interaction. The number of 'liberated' partons will depend on the hard scale $Q$ : the larger the value of $Q$, the more sudden the deflection of the struck parton, and the fewer the partons that can reconnect before its departure (typically only partons with virtuality larger than $Q$ ).

After the hard process, the partons liberated during the evolution prior to the collision and the partons created by the hard collision will themselves emit radiation. The radiation process, governed by perturbative QCD, continues until a low virtuality scale is reached (the boundary region labelled with a dotted line, $\mathrm{H}$, in our figure). To describe this perturbative evolution phase, proper care has to be taken to incorporate quantum coherence effects, which in principle connect the probabilities of radiation off different partons in the event. Once the low virtuality scale is reached, the memory of the hard-process phase has been lost, once again as a result of different time scales in the problem, and the final phase of hadronization takes over. Because of the decoupling from the hard-process phase, the hadronization is assumed to be independent of the initial hard process, and its parametrization, tuned to the observables of some reference process, can then be used in other hard interactions (universality of hadronization). Nearby partons merge into colour-singlet clusters (the grey blobs in fig. 1), which are then decayed phenomenologically into physical hadrons. To complete the picture, we need to understand the evolution of the fragments of the initial hadrons. As shown in the figure, this evolution cannot be entirely independent of what happens in the hard event, because at least colour quantum numbers must be exchanged to guarantee the overall neutrality and conservation of baryon number. In our example, the gluons $f$ and $g$, emitted early on in the perturbative evolution of the initial state, split into $q \bar{q}$ pairs which are shared between the hadron fragments (whose overall interaction is represented by the oval labelled UE, for Underlying Event) and the clusters resulting from the evolution of the initial state.

The above ideas are embodied in the following factorization formula, which represents the starting point of any theoretical analysis of cross-sections and observables in hadronic collisions:

$$
\frac{d \sigma}{d X}=\sum_{j, k} \int_{\hat{X}} f_{j}\left(x_{1}, Q_{i}\right) f_{k}\left(x_{2}, Q_{i}\right) \frac{d \hat{\sigma}_{j k}\left(Q_{i}, Q_{f}\right)}{d \hat{X}} F\left(\hat{X} \rightarrow X ; Q_{i}, Q_{f}\right),
$$

where:

- $X$ is some hadronic observable (e.g. the transverse momentum of a pion, the energy of a jet, the invariant mass of a combination of particles, etc.);

- the sum over $j$ and $k$ extends over the partons types inside the colliding hadrons;

- the function $f_{j}(x, Q)$ (known as parton distribution function, PDF) represents the number density of parton type $j$ with momentum fraction $x$ in a proton probed at a scale $Q_{i}$ (more later on the meaning of this scale); 
- $\hat{X}$ is a parton-level kinematical variable (e.g. the transverse momentum of a parton from the hard scattering);

- $\hat{\sigma}_{j k}$ is the parton-level cross-section, differential in the variable $\hat{X}$;

- $F\left(\hat{X} \rightarrow X ; Q_{i}, Q_{f}\right)$ is a transition function, weighting the probability that the partonic state defining $\hat{X}$ gives rise, after hadronization, to the hadronic observable $X$;

- the scales $Q_{i}$ and $Q_{f}$ correspond to the scales at which we separate the hard, perturbative, process from the initial and final-state evolutions, respectively.

In the rest of this Section I shall cover the above ideas in some more detail. While I shall not provide you with a rigorous proof of the legitimacy of this approach, I shall try to justify it qualitatively to make it sound at least plausible.

\subsection{The parton densities and their evolution}

As mentioned above, the binding forces responsible for the quark confinement are due to the exchange of rather soft gluons. If a quark were to exchange just a single a hard virtual gluon with another quark, the recoil would tend to break the proton apart. It is easy to verify that the exchange of gluons with virtuality larger than $Q$ is then proportional to some large power of $m_{p} / Q, m_{p}$ being the proton mass. Since the gluon coupling constant gets smaller at large $Q$, exchange of hard gluons is significantly suppressed ${ }^{1}$. Consider in fact the picture in Fig. 2. The exchange of two gluons is required to ensure that the mo-

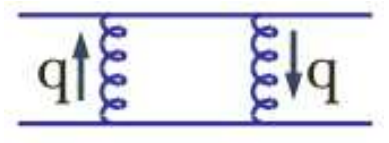

Fig. 2: Gluon exchange inside the proton

mentum exchanged after the first gluon emission is returned to the quark, and the proton maintains its structure. The contributions of hard gluons to this process can be approximated by integrating the loop over large momenta:

$$
\int_{Q} \frac{d^{4} q}{q^{6}} \sim \frac{1}{Q^{2}}
$$

At large $Q$ this contribution is suppressed by powers of $\left(m_{p} / Q\right)^{2}$, where the proton mass $m_{p}$ is included as being the only dimensionful quantity available (one could use here the fundamental scale of QCD, $\Lambda_{Q C D}$, but numerically this is anyway of the order of a GeV). The interactions keeping the proton together are therefore dominated by soft exchanges, with virtuality $Q$ of the order of $m_{p}$. Owing to Heisenberg's uncertainty principle, the typical time scale of these exchanges is of the order of $1 / m_{p}$ : this is the time during which fluctuations with virtuality of the order of $m_{p}$ can survive. In the laboratory system, where the proton travels with energy $E$, this time is Lorentz dilated to $\tau \sim \gamma / m_{p}=E / m_{p}^{2}$. If we probe the proton with an off-shell photon, the interaction takes place during the limited lifetime of the virtual photon, which, once more from the uncertainty principle, is given by the inverse of its virtuality. Assuming the virtuality $Q \gg m_{p}$, once the photon gets 'inside' the proton and meets a quark, the struck quark has no time to negotiate a coherent response with the other quarks, because the time scale for it to 'talk' to its partners is too long compared with the duration of the interaction with the photon itself. As a result, the struck quark has no option but to interact with the photon as if it were a free particle. Let us look in more detail at what happens during such a process. In Fig. 3 we see a

\footnotetext{
${ }^{1}$ The fact that the coupling decreases at large $Q$ plays a fundamental role in this argument. Were this not true, the parton picture could not be used!.
} 


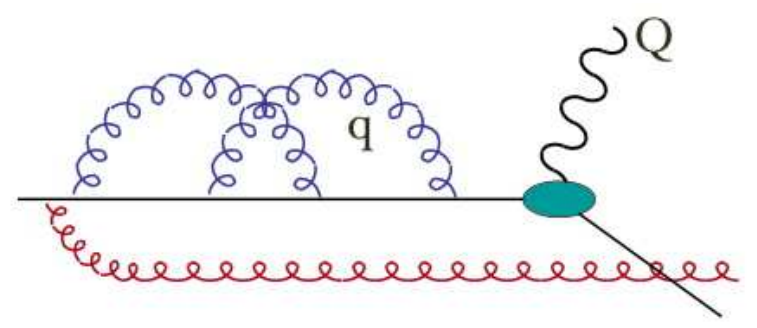

Fig. 3: Gluon emission at different scales during the approach to a hard collision.

proton as it approaches a hard collision with a photon of virtuality $Q$. Gluons emitted at a scale $q>Q$ have the time to be reabsorbed, since their lifetime is very short. Their contribution to the process can be calculated in perturbative QCD, since the scale is large and in the domain where perturbative calculations are meaningful. Since after being reabsorbed the state of the quark remains the same, their only effect is an overall renormalization of the wave function, and they do not affect the quark density. A gluon emitted at a scale $q<Q$, however, has a lifetime longer than the time it takes for the quark to interact with the photon, and by the time it tries to reconnect to its parent quark, the quark has been kicked away by the photon, and is no longer there. Since the gluon has taken away some of the quark momentum, the momentum fraction $x$ of the quark as it enters the interaction with the photon is different than the momentum it had before, and therefore its density $f(x)$ is affected. Furthermore, when the scale $q$ is of the order of $1 \mathrm{GeV}$ the state of the quark is not calculable in perturbative QCD. This state depends on the internal wave function of the proton, which perturbative QCD cannot easily predict. We can, however, say that the wave function of the proton, and therefore the state of the 'free' quark, are determined by the dynamics of the soft-gluon exchanges inside the proton itself. Since the time scale of this dynamics is long relative to the time scale of the photon-quark interaction, we can safely argue that the photon sees to good approximation a static snapshot of the proton's inner guts. In other words, the state of the quark had been prepared long before the photon arrived. This also suggests that the state of the quark will not depend on the precise nature of the external probe, provided the time scale of the hard interaction is very short compared to the time it would take for the quark to readjust itself. As a result, if we could perform some measurement of the quark state using, say, a virtual-photon probe, we could then use this knowledge on the state of the quark to perform predictions for the interaction of the proton with any other probe (e.g. a virtual $W$ or even a gluon from an opposite beam of hadrons). This is the essence of the universality of the parton distributions.

The above picture leads to an important observation. It appears in fact that the distinction between which gluons are reabosrbed and which ones are not depends on the scale $Q$ of the hard probe. As a result, the parton density $f(x)$ appears to depend on $Q$. This is illustrated in Fig. 4. The gluon emitted

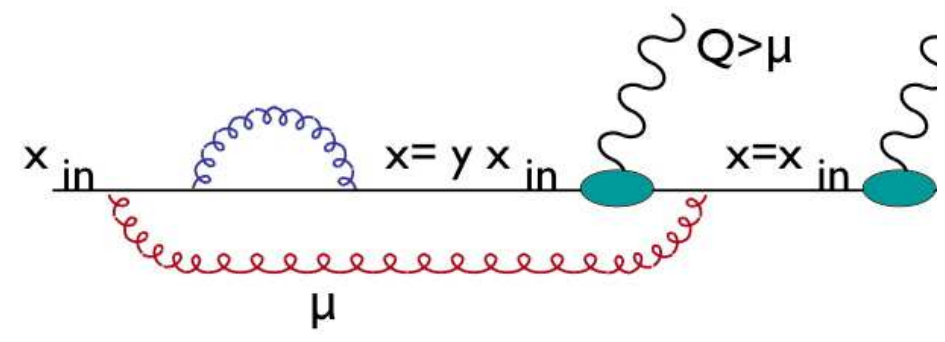

Fig. 4: Scale dependence of the gluon emission during a hard collision

at a scale $\mu$ has a lifetime short enough to be reabsorbed before a collision with a photon of virtuality 
$Q<\mu$, but too long for a photon of virtuality $Q>\mu$. When going from $\mu$ to $Q$, therefore, the partonic density $f(x)$ changes. We can easily describe this variation as follows:

$$
f(x, Q)=f(x, \mu)+\int_{x}^{1} d x_{i n} f\left(x_{i n}, \mu\right) \int_{\mu}^{Q} d q^{2} \int_{0}^{1} d y \mathcal{P}\left(y, q^{2}\right) \delta\left(x-y x_{i n}\right),
$$

Here we obtain the density at the scale $Q$ by adding to $f(x)$ at the scale $\mu$ (which we label as $f(x, \mu)$ ) all the quarks with momentum $x_{i n}>x$ that retain a proton-momentum fraction $x=y / x_{i n}$ by emitting a gluon. The function $P\left(y, Q^{2}\right)$ describes the 'probability' that the quark emits a gluon at a scale $Q$, keeping a fraction $y$ of its momentum. This function does not depend on the details of the hard process, it simply describes the radiation of a free quark subject to an interaction with virtuality $Q$. Since $f(x, Q)$ does not depend upon $\mu$ ( $\mu$ is just used as a reference scale to construct our argument), the total derivative of the right-hand side w.r.t. $\mu$ should vanish, leading to the following equation:

$$
\frac{d f(x, Q)}{d \mu^{2}}=0 \Rightarrow \frac{d f(x, \mu)}{d \mu^{2}}=\int_{x}^{1} \frac{d y}{y} f(y, \mu) \mathcal{P}\left(x / y, \mu^{2}\right) .
$$

With additional considerations and explicit calculations, one can prove that

$$
\mathcal{P}\left(x, Q^{2}\right)=\frac{\alpha_{s}}{2 \pi} \frac{1}{Q^{2}} P(x)
$$

from which the Dokshitzer-Gribov-Lipatov-Altarelli-Parisi (DGLAP) equation follows [14-16]:

$$
\frac{d f(x, \mu)}{d \log \mu^{2}}=\frac{\alpha_{s}}{2 \pi} \int_{x}^{1} \frac{d y}{y} f(y, \mu) P_{q q}(x / y) .
$$

The so-called splitting function $P_{q q}(x)$ can be calculated in perturbative QCD. The subscript $q q$ is a labelling convention indicating that $x$ refers to the momentum fraction retained by a quark after emission of a gluon.
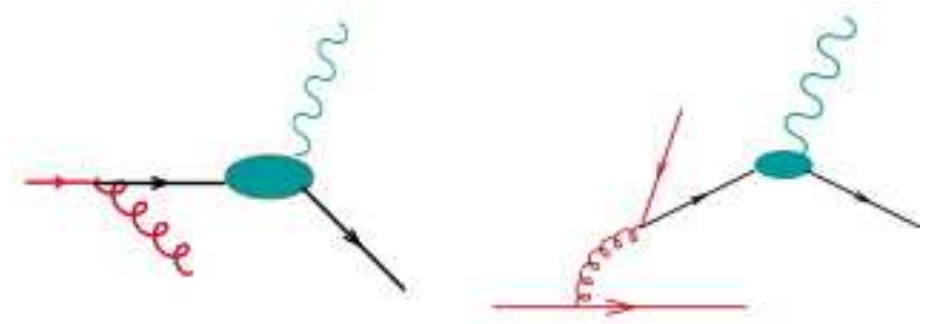

Fig. 5: The processes leading to the evolution of the quark density

More generally, one should consider additional processes. For example, one should include cases in which the quark interacting with the photon comes from the splitting of a gluon. This is shown in Fig. 5: the left diagram is the one we considered above; the right diagram corresponds to processes where an emitted gluon has the time to split into a $q \bar{q}$ pair, and it is one of these quarks which interacts with the photon. The overall evolution equation, including the effect of gluon splitting, is given by

$$
\frac{d q(x, Q)}{d t}=\frac{\alpha_{s}}{2 \pi} \int_{x}^{1} \frac{d y}{y}\left[q(y, Q) P_{q q}\left(\frac{x}{y}\right)+g(y, Q) P_{q g}\left(\frac{x}{y}\right)\right],
$$

where $t=\log Q^{2}$. For external probes that couple to gluons (for example an external gluon, coming e.g. from an incoming proton), we have a similar evolution of the gluon density (see Fig. 6):

$$
\frac{d g(x, Q)}{d t}=\frac{\alpha_{s}}{2 \pi} \int_{x}^{1} \frac{d y}{y}\left[g(y, Q) P_{g g}\left(\frac{x}{y}\right)+\sum_{q, \bar{q}} q(y, Q) P_{g q}\left(\frac{x}{y}\right)\right] \text {. }
$$



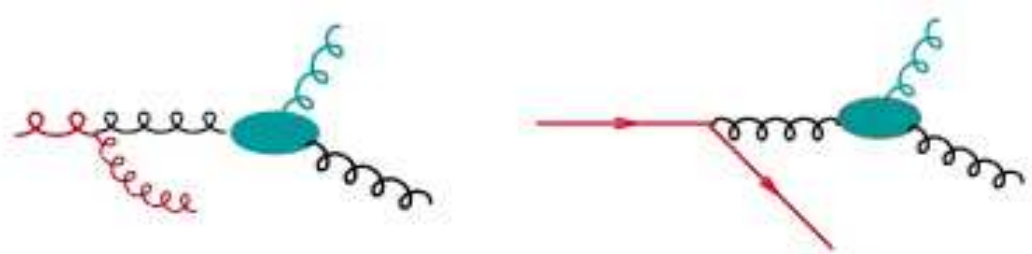

Fig. 6: The processes leading to the evolution of the gluon density

The explicit calculation of the splitting functions $P_{i j}(x)$ (see, for example, Ref. [12]) gives then the following expressions ${ }^{2}$ :

$$
\begin{aligned}
P_{q q}(x) & =P_{g q}(1-x)=C_{F} \frac{1+x^{2}}{1-x} \\
P_{q g}(x) & =\frac{1}{2}\left[x^{2}+(1-x)^{2}\right] \\
P_{g g}(x) & =2 C_{A}\left[\frac{1-x}{x}+\frac{x}{1-x}+x(1-x)\right],
\end{aligned}
$$

where $C_{F}=\left(N_{C}^{2}-1\right) / 2 N_{C}$ and $C_{A}=2 N_{C}$ are the Casimir invariants of the fundamental and adjoint representation of $S U\left(N_{C}\right)\left(N_{C}=3\right.$ for QCD). In the following we shall derive some general properties of the PDF evolution, and give a few concrete examples.

\subsection{General properties of parton density evolution}

Defining the moments of an arbitrary function $g(x)$ as follows:

$$
g_{n}=\int_{0}^{1} \frac{d x}{x} x^{n} g(x)
$$

it is easy to prove that the evolution equations for the moments turn into ordinary linear differential equations:

$$
\begin{aligned}
\frac{d f_{i}^{(n)}}{d t} & =\frac{\alpha_{s}}{2 \pi}\left[P_{q q}^{(n)} f_{i}^{(n)}+P_{q g}^{(n)} f_{g}^{(n)}\right] \\
\frac{d f_{g}^{(n)}}{d t} & =\frac{\alpha_{s}}{2 \pi}\left[P_{g g}^{(n)} f_{g}+P_{g q}^{(n)} f_{i}^{(n)}\right] .
\end{aligned}
$$

It is convenient to introduce the concepts of valence $(V(x, t))$ and singlet $(\Sigma(x, t))$ densities:

$$
\begin{aligned}
V(x) & =\sum_{i} f_{i}(x)-\sum_{\bar{\imath}} f_{\bar{\imath}}(x), \\
\Sigma(x) & =\sum_{i} f_{i}(x)+\sum_{\bar{\imath}} f_{\bar{\imath}}(x),
\end{aligned}
$$

where the index $\bar{\imath}$ refers to the antiquark flavours. The evolution equations then become

$$
\frac{d V^{(n)}}{d t}=\frac{\alpha_{s}}{2 \pi} P_{q q}^{(n)} V^{(n)},
$$

\footnotetext{
${ }^{2}$ The expressions given here are strictly valid only for $x \neq 1$. The slight modifications required to extend them to $x=1$ will be justified and introduced in the next section.
} 


$$
\begin{aligned}
\frac{d \Sigma^{(n)}}{d t} & =\frac{\alpha_{s}}{2 \pi}\left[P_{q q}^{(n)} \Sigma^{(n)}+2 n_{f} P_{q g}^{(n)} f_{g}^{(n)}\right] \\
\frac{d f_{g}^{(n)}}{d t} & =\frac{\alpha_{s}}{2 \pi}\left[P_{g q}^{(n)} \Sigma^{(n)}+P_{g g}^{(n)} f_{g}^{(n)}\right] .
\end{aligned}
$$

Note that the equation for the valence density decouples from the evolution of the gluon and singlet densities, which are coupled among themselves. This is physically very reasonable, since in perturbation theory the contribution to the quark and the antiquark densities coming form the evolution of gluons (via their splitting into $q \bar{q}$ pairs) is the same, and will cancel out in the definition of the valence. The valence therefore only evolves because of gluon emission. On the contrary, gluons and $q \bar{q}$ pairs in the proton sea evolve into one another.

The first moment of $V(x), V^{(1)}=\int_{0}^{1} d x V(x)$, counts the number of valence quarks. We therefore expect it to be independent of $Q^{2}$ :

$$
\frac{d V^{(1)}}{d t} \equiv 0=\frac{\alpha_{s}}{2 \pi} P_{q q}^{(1)} V^{(1)}=0 .
$$

Since $V^{(1)}$ itself is different from 0 , we obtain a constraint on the first moment of the splitting function: $P_{q q}^{(1)}=0$. This constraint is satisfied by including the effect of the virtual corrections, which generate a contribution to $P_{q q}(z)$ proportional to $\delta(1-z)$. This correction is incorporated in $P_{q q}(z)$ via the redefinition

$$
P_{q q}(z) \rightarrow\left(\frac{1+z^{2}}{1-z}\right)_{+} \equiv \frac{1+z^{2}}{1-z}-\delta(1-z) \int_{0}^{1} d y\left(\frac{1+y^{2}}{1-y}\right)
$$

where the + sign turns $P_{q q}(z)$ into a distribution. In this way, $\int_{0}^{1} d z P_{q q}(z)=0$ and the valence sum-rule is obeyed at all $Q^{2}$.

Another sum rule which does not depend on $Q^{2}$ is the momentum sum-rule, which imposes the constraint that all of the momentum of the proton be carried by its constituents (valence plus sea plus gluons):

$$
\int_{0}^{1} d x x\left[\sum_{i, \bar{i}} f_{i}(x)+f_{g}(x)\right] \equiv \Sigma^{(2)}+f_{g}^{(2)}=1 .
$$

Once more this relation should hold for all $Q^{2}$ values, and you can prove by using the evolution equations that this implies

$$
\begin{aligned}
P_{q q}^{(2)}+P_{g q}^{(2)} & =0, \\
P_{g g}^{(2)}+2 n_{f} P_{q g}^{(2)} & =0 .
\end{aligned}
$$

You can check using the definition of second moment, and the explicit expressions of the $P_{q q}$ and $P_{g q}$ splitting functions, that the first condition is automatically satisfied. The second condition is satisfied by including the virtual effects in the gluon propagator, which contribute a term proportional to $\delta(1-z)$. It is a simple exercise to verify that the final form of the $P_{g g}(z)$ splitting function, satisfying Eq. (23), is

$$
P_{g g} \rightarrow 2 C_{A}\left\{\frac{x}{(1-x)_{+}}+\frac{1-x}{x}+x(1-x)\right\}+\delta(1-x)\left[\frac{11 C_{A}-2 n_{f}}{6}\right] .
$$

\subsection{Solution of the evolution equations}

The evolution equations formulated in the previous section can be solved analytically in moment space. The boundary conditions are given by the moments of the parton densities at a given scale $\mu$, where, in principle, they can be obtained from a direct measurement. The solution at different values of the scale $Q$ can then be obtained by inverting numerically the expression for the moments back to $x$ space. The 
resulting evolved densities can then be used to calculate cross-sections for an arbitrary process involving hadrons, at an arbitrary scale $Q$. We shall limit ourselves here to studying some properties of the analytic solutions, and will present and comment on some plots obtained from numerical studies available in the literature.

As an exercise, you can show that the solution of the evolution equation for the valence density is the following:

$$
V^{(n)}\left(Q^{2}\right)=V^{(n)}\left(\mu^{2}\right)\left[\frac{\log Q^{2} / \Lambda^{2}}{\log \mu^{2} / \Lambda^{2}}\right]^{P_{q q}^{(n)} / 2 \pi b_{0}}=V^{(n)}\left(\mu^{2}\right)\left[\frac{\alpha_{s}\left(\mu^{2}\right)}{\alpha_{s}\left(Q^{2}\right)}\right]^{P_{q q}^{(n)} / 2 \pi b_{0}}
$$

where the running of $\alpha_{s}\left(\mu^{2}\right)$ has to be taken into account to get the right result. Since all moments $P^{(n)}$ are negative, the evolution to larger values of $Q$ makes the valence distribution softer and softer. This is physically reasonable, since the only thing that the valence quarks can do is to lose energy because of gluon emission.

The solutions for the gluon and singlet distributions $f_{g}$ and $\Sigma$ can be obtained by diagonalizing the $2 \times 2$ system in Eqs. (17) and (18). We study the case of the second moments, which correspond to the momentum fractions carried by quarks and gluons separately. In the asymptotic limit, $\Sigma^{(2)}$ goes to a constant, and $\frac{d \Sigma^{(2)}}{d t}=0$. Then, using the momentum sum rule:

$$
\begin{aligned}
P_{q q}^{(2)} \Sigma^{(2)}+2 n_{f} P_{q g}^{(2)} f_{g}^{(2)} & =0 \\
\Sigma^{(2)}+f_{g}^{(2)} & =1
\end{aligned}
$$

The solution of this system is

$$
\begin{gathered}
\Sigma^{(2)}=\frac{1}{1+\frac{4 C_{F}}{n_{f}}} \quad\left(=15 / 31 \text { for } n_{f}=5\right) \\
f_{g}^{(2)}=\frac{4 C_{F}}{4 C_{F}+n_{f}} \quad\left(=16 / 31 \text { for } n_{f}=5\right) .
\end{gathered}
$$

As a result, the fraction of momentum carried by gluons is asymptotically approximately $50 \%$ of the total proton momentum. It is interesting to note that, experimentally, this asymptotic value is actually reached already at rather low values of $Q^{2}$. It was indeed observed already in the first deep-inelastic ep experiments, which exposed the possible presence of quarks in the proton, that only approximately $50 \%$ of the proton momentum was carried by charged constituents. This was one of the early pieces of evidence for the existence of gluons.

\subsection{Example: quantitative evolution of parton densities}

As I mentioned earlier, a complete solution for the evolved parton densities in $x$ space can only be obtained from a numerical analysis. This work has been done in the past by several groups (see, for example, the chapter on PDFs contained in Ref. [8]), and is continuously being updated by including the most up-to-date experimental results used for the determination of the input densities at a fixed scale. The left side of fig. 7 shows the up-quark valence momentum density at various scales $Q$. Note the softening at larger scales, and the clear $\log Q^{2}$ evolution. As $Q^{2}$ grows, the valence quarks emit more and more radiation, since they change direction over a shorter amount of time (larger acceleration). They therefore lose more momentum to the emitted gluons, and their spectrum becomes softer. The most likely momentum fraction carried by a valence up quark in the proton goes from $x \sim 20 \%$ at $Q=3 \mathrm{GeV}$, to $x \lesssim 10 \%$ at $Q=1000 \mathrm{GeV}$. Notice finally that the density vanishes at small $x$.

The right-hand side of fig. 7 shows the gluon momentum density. This grows at small $x$, with an approximate $g(x) \sim 1 / x^{1+\delta}$ behaviour, and $\delta>0$ slowly increasing at large $Q^{2}$. This low- $x$ growth 

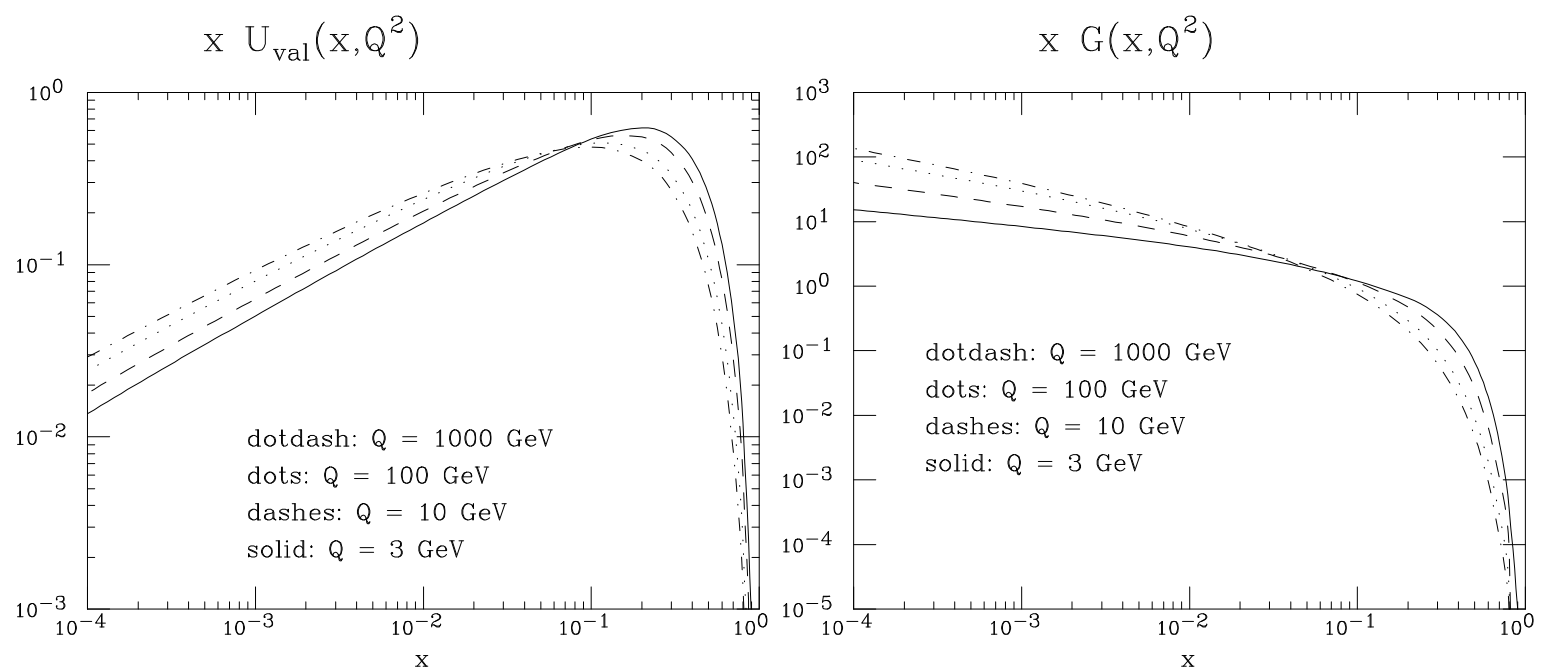

Fig. 7: Left: Valence up-quark momentum-density distribution, for different scales $Q$. Right: Gluon momentumdensity distribution.

is due to the $1 / x$ emission probability for the radiation of gluons, which was discussed in the previous lecture and which is represented by the $1 / x$ factors in the $P_{g q}(x)$ and $P_{g g}(x)$ splitting functions. As $Q^{2}$ grows we find an increasing number of gluons at small $x$, as a result of the increased radiation off quarks, as well as off the harder gluons.
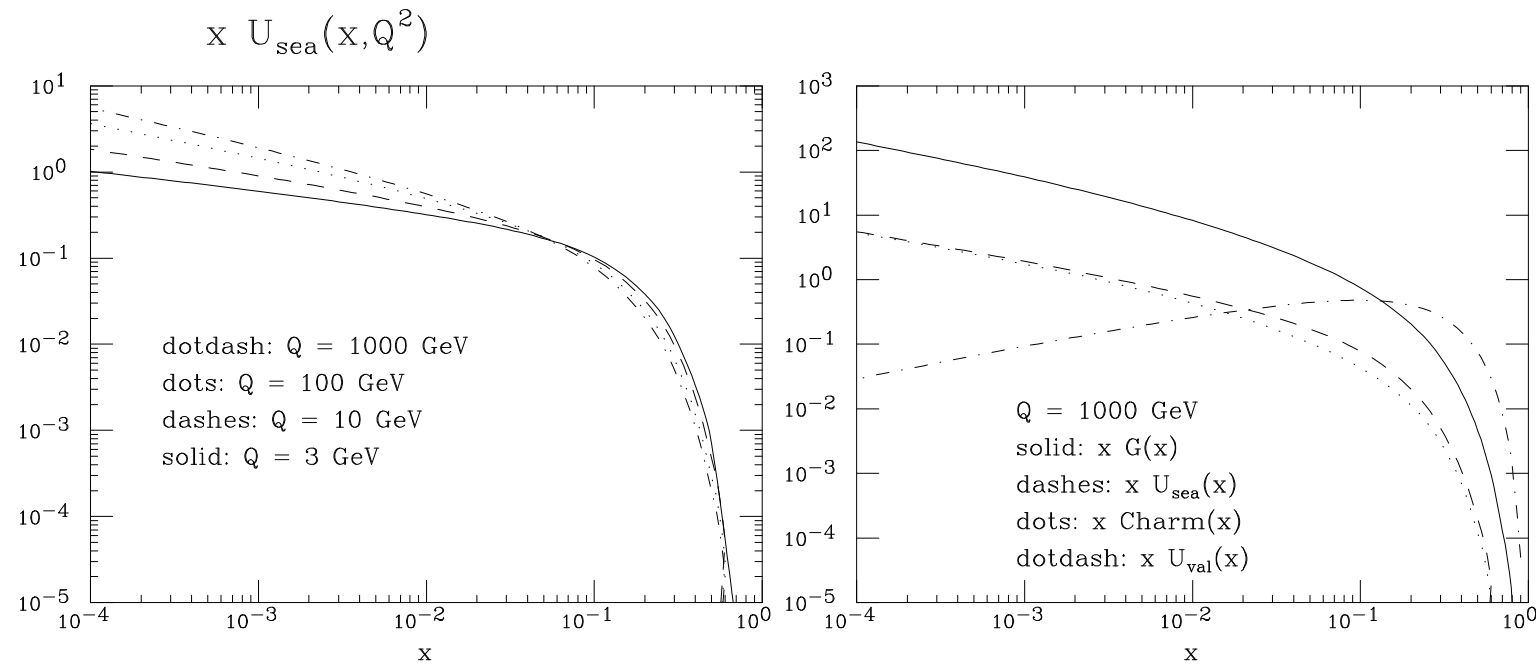

Fig. 8: Left: Sea up-quark momentum-density distribution, for different scales $Q$. Right: Momentum-density distribution for several parton species, at $Q=1000 \mathrm{GeV}$.

The left-hand side of Fig. 8 shows the evolution of the up-quark sea momentum density. Shape and evolution match those of the gluon density, a consequence of the fact that sea quarks come from the splitting of gluons. Since the gluon-splitting probability is proportional to $\alpha_{s}$, the approximate ratio sea/gluon $\sim 0.1$ which can be obtained by comparing Figs. 7 and 8 is perfectly justified.

Finally, the momentum densities for gluons, up-sea, charm, and up-valence distributions are shown, for $Q=1000 \mathrm{GeV}$, on the right side of Fig.8. Note here that $u_{\text {sea }}$ and charm are approximately the same at very large $Q$ and small $x$, as will be discussed in more detail in the next subsection. 
The proton momentum is mostly carried by valence quarks and by gluons. The contribution of sea quarks is negligible.

Parton densities are extracted from experimental data. Their determination is therefore subject to the statistical and systematic uncertainties of the experiments and of the theoretical analysis (e.g. the treatment of non-perturbative effects, the impact of missing higher-order perturbative corrections). Techniques have been introduced recently to take into account these uncertainties, and to evaluate their impact on concrete observables. A summary of such an analysis, for the LHC, is given in Fig. 9. What is plotted is the uncertainty bands for partonic luminosities ${ }^{3}$ corresponding to the $g g$ and $q \bar{q}$ initial-state channels. The partonic flux is given as a function of $\hat{s}$, the partonic CM invariant mass. Obvious features include the growth of uncertainty of the $g g$ density at large mass, corresponding to the lack of data covering the large- $x$ region of the gluon density. Notice that the $g g$ luminosity drives the production of both $t \bar{t}$ and Higgs production, at mass values around 350 and $125 \mathrm{GeV}$, respectively. In this mass range, the PDF uncertainty is today at the level of 2-3\%. For the $q \bar{q}$, which drives the production of DY pairs (or of new vector gauge bosons), the uncertainty is likewise in the few percent range up to masses of about $1 \mathrm{TeV}$, and remains below $20 \%$ even up to $4 \mathrm{TeV}$.
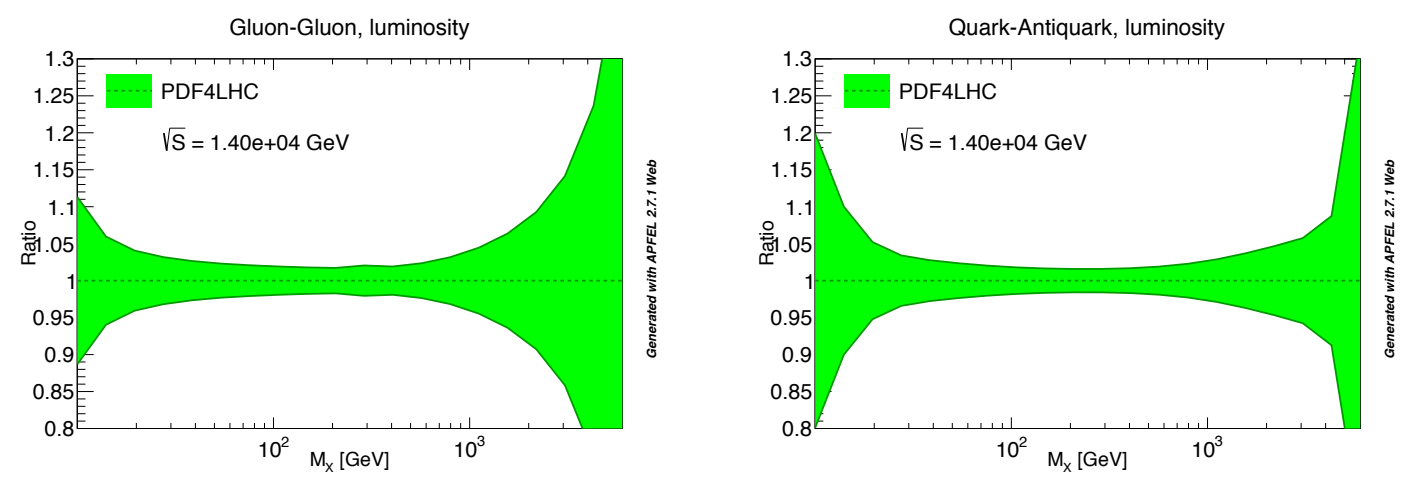

Fig. 9: Uncertainty in the parton luminosity functions at the LHC, using the PDF4LHC set of PDFs [9]. The plot was obtained using the Apfel web resource $[10,11]$.

\subsection{Example: the charm content of the proton}

If the virtuality of the external probe is large enough, the time scale of the hard interaction is so short that gluon fluctuations into virtual heavy quark states can be directly exposed, and the virtual heavy quarks (charm quarks in our example) can be brought on-shell via the interaction with the photon (see fig. 10). To the external photon, it will therefore appear as if the proton contained some charm. While in the

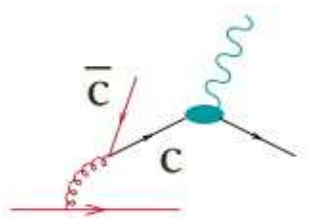

Fig. 10: Gluon evolution leading to a charm quark content of the proton

case of the gluons and of light quarks the boundary condition for the DGLAP evolution at small $Q$ is non-perturbative and cannot be derived from first principles, in the case of a heavy quark $Q$ the boundary condition $f_{Q}\left(x, Q_{0}\right)=0$ holds at a scale $Q_{0} \sim m_{Q}$ that is large enough for perturbation theory to apply.

\footnotetext{
${ }^{3}$ For the definition of parton luminosity see Section 4.1 .
} 
The charm density can be calculated assuming that the heavy quark density itself is 0 at $Q \sim m_{c}$, and builds up according to the DGLAP evolution equation:

$$
\frac{d c(x, Q)}{d t}=\frac{\alpha_{s}}{2 \pi} \int_{x}^{1} \frac{d y}{y} g(y, Q) P_{q g}\left(\frac{x}{y}\right) .
$$

Assuming a gluon density behaving like $g(x, Q) \sim A / x$, which is a first approximation to a bremstrahlung spectrum, we can easily calculate

$$
\begin{aligned}
\frac{d c(x, Q)}{d t} & =\frac{\alpha_{s}}{2 \pi} \int_{x}^{1} \frac{d y}{y} g(x / y, Q) P_{q g}(y)=\frac{\alpha_{s}}{2 \pi} \int_{x}^{1} d y \frac{A}{x} \frac{1}{2}\left[y^{2}+(1-y)^{2}\right] \\
& =\frac{\alpha_{s}}{6 \pi} \frac{A}{x} c(x, Q) \sim \frac{\alpha_{s}}{6 \pi} \log \left(\frac{Q^{2}}{m_{c}^{2}}\right) g(x, Q) .
\end{aligned}
$$

The charm density is therefore proportional to the gluon density, up to an overall factor proportional to $\alpha_{s}$. When $Q$ becomes very large, the effect of the quark mass becomes subleading, and we expect all sea quarks to reach asymptotically the same density.

While this is a simplified approach to the estimate of the heavy quark density of the proton, the approximation is rather good. This is shown by the plots in fig. 11, which compare the charm and bottom PDF as given by Eq. 31 with the result extracted from a full set of PDFs. The solid histograms in these plots represent the exact result, for three values of the evolution scale $Q$. The diamonds give the approximate results. Notice that the agreement is very good at small $x$ and at the smaller values of $Q$. At larger $x$ the approximation deteriorates, since in that case the assumption that $g(x) \sim 1 / x$ is no longer valid. At higher scales $Q$ the exact result becomes smaller than the approximate one, since the latter neglects the momentum loss due to the higher-order gluon radiation (namely the contributions to the evolution equation proportional to $P_{q q}(y) \times Q(x / y)$ ). Of course, any accurate calculation of crosssections involving initial-state heavy quarks will make use of the exact results, but it is interesting to see that even in such a complex process it is possible to identify useful analytic approximations that can give us good order-of-magnitude estimates!
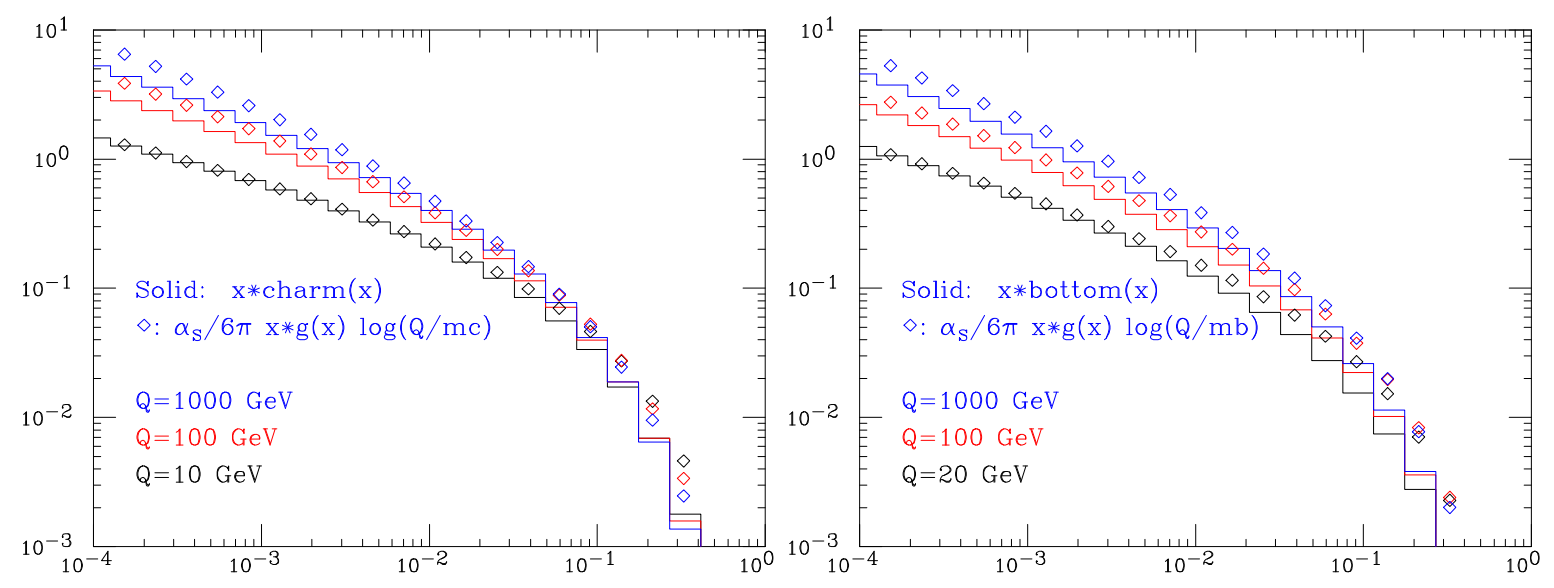

Fig. 11: Charm and bottom quark PDFs, as obtained from the exact and approximate evolutions.

\section{The evolution of quarks and gluons}

We discussed in the previous section the initial-state evolution of quarks and gluons as the proton approaches the hard collision. We study here how quarks and gluons evolve, and finally transform into hadrons, neutralizing their colours. We start by considering the simplest case: $e^{+} e^{-}$collisions, which 
provide the cleanest environment in which to study applications of QCD at high energy. This is the place where theoretical calculations have today reached their best accuracy, and where experimental data are the most precise, especially thanks to the huge statistics accumulated by LEP, LEP2 and SLC. The key process is the annihilation of the $e^{+} e^{-}$pair into a virtual photon or $Z^{0}$ boson, which will subsequently decay to a $q \bar{q}$ pair. $e^{+} e^{-}$collisions have therefore the big advantage of providing an almost point-like source of quark pairs, so that, in contrast to the case of interactions involving hadrons in the initial state, we at least know very precisely the state of the quarks at the beginning of the interaction process.

Nevertheless, it is by no means obvious that this information is sufficient to predict the properties of the hadronic final state. We know that this final state is clearly not simply a $q \bar{q}$ pair, but some highmultiplicity set of hadrons. For example, as shown in Fig. 12, the average multiplicity of charged hadrons in the decay of a $Z^{0}$ is approximately 20 . It is therefore not obvious that a calculation done using the

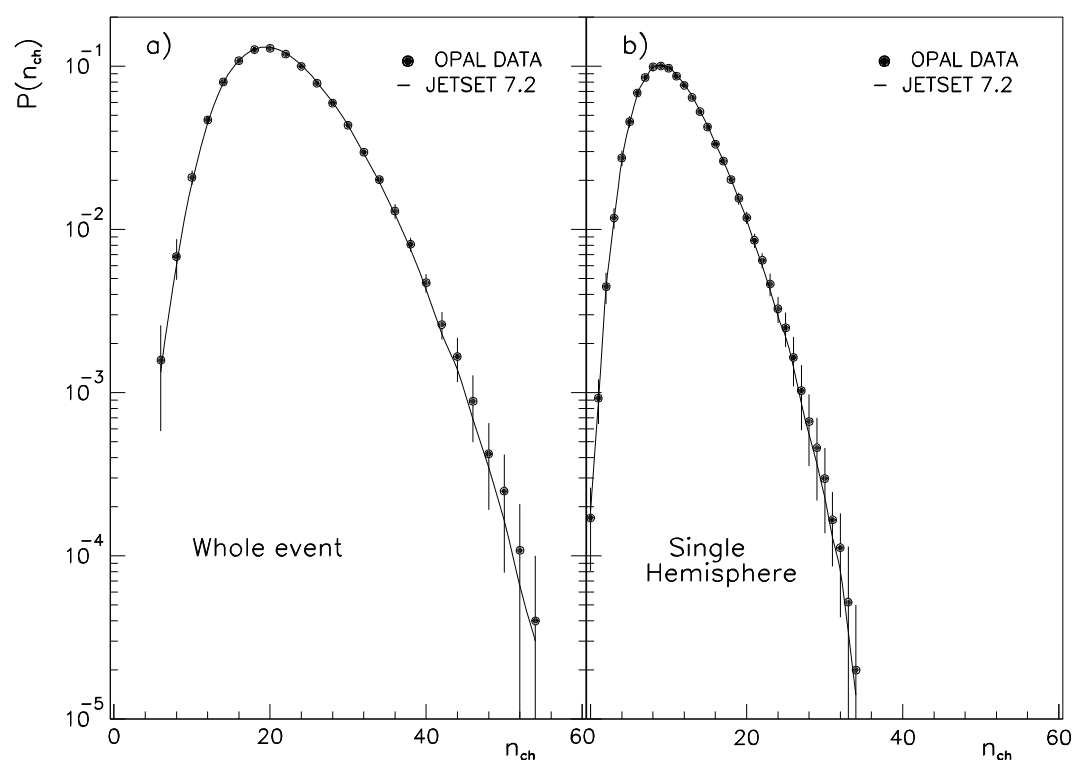

Fig. 12: Charged particle multiplicity distribution in $Z^{0}$ decays

simple picture $e^{+} e^{-} \rightarrow q \bar{q}$ (see Fig. 13) has anything to do with reality. For example, one may wonder

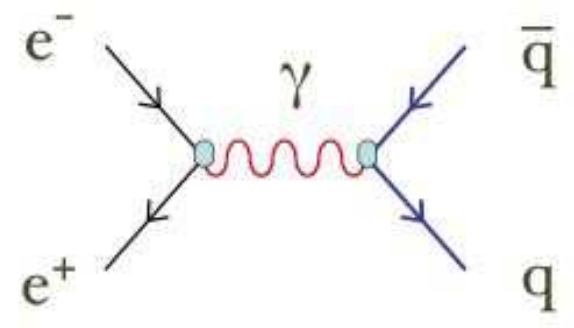

Fig. 13: Tree level production of a $q \bar{q}$ pair in $e^{+} e^{-}$collisions

why we do not need to calculate $\sigma\left(e^{+} e^{-} \rightarrow q \bar{q} g \ldots g \ldots\right)$ for all possible gluon multiplicities to get an accurate estimate of $\sigma\left(e^{+} e^{-} \rightarrow\right.$ hadrons $)$. And since in any case the final state is not made of $q$ 's and $g$ 's, but of $\pi$ 's, $K$ 's, $\rho$ 's, etc., why would $\sigma\left(e^{+} e^{-} \rightarrow q \bar{q} g \ldots g\right)$ be enough?

The solution to this puzzle lies both in a question of time and energy scales, and in the dynamics of QCD. When the $q \bar{q}$ pair is produced, the force binding $q$ and $\bar{q}$ is proportional to $\alpha_{s}(s)$ ( $\sqrt{s}$ being 
the $e^{+} e^{-}$centre-of-mass energy). Therefore it is weak, and $q$ and $\bar{q}$ behave to good approximation like free particles. The radiation emitted in the first instants after the pair creation is also perturbative, and it will stay so until a time after creation of the order of $(1 \mathrm{GeV})^{-1}$, when radiation with wavelengths $\gtrsim(1 \mathrm{GeV})^{-1}$ starts being emitted. At this scale the coupling constant is large, and non-perturbative phenomena and hadronization start playing a rôle. However, as we shall show, colour emission during the perturbative evolution organizes itself in such a way as to form colour-neutral, low-mass, parton clusters highly localized in phase-space. As a result, the complete colour-neutralization (i.e., the hadronization) does not involve long-range interactions between partons far away in phase-space. This is very important, because the forces acting among coloured objects at this time scale would be huge. If the perturbative evolution were to separate far apart colour-singlet $q \bar{q}$ pairs, the final-state interactions taking place during the hadronization phase would totally upset the structure of the final state.

In this picture, the identification of the perturbative cross-section $\sigma\left(e^{+} e^{-} \rightarrow q \bar{q}\right)$ with observable, high-multiplicity hadronic final states is realised by jets, namely collimated streams of hadrons that are the final result of the perturbative and non-perturbative evolution of each quark. The large multiplicity
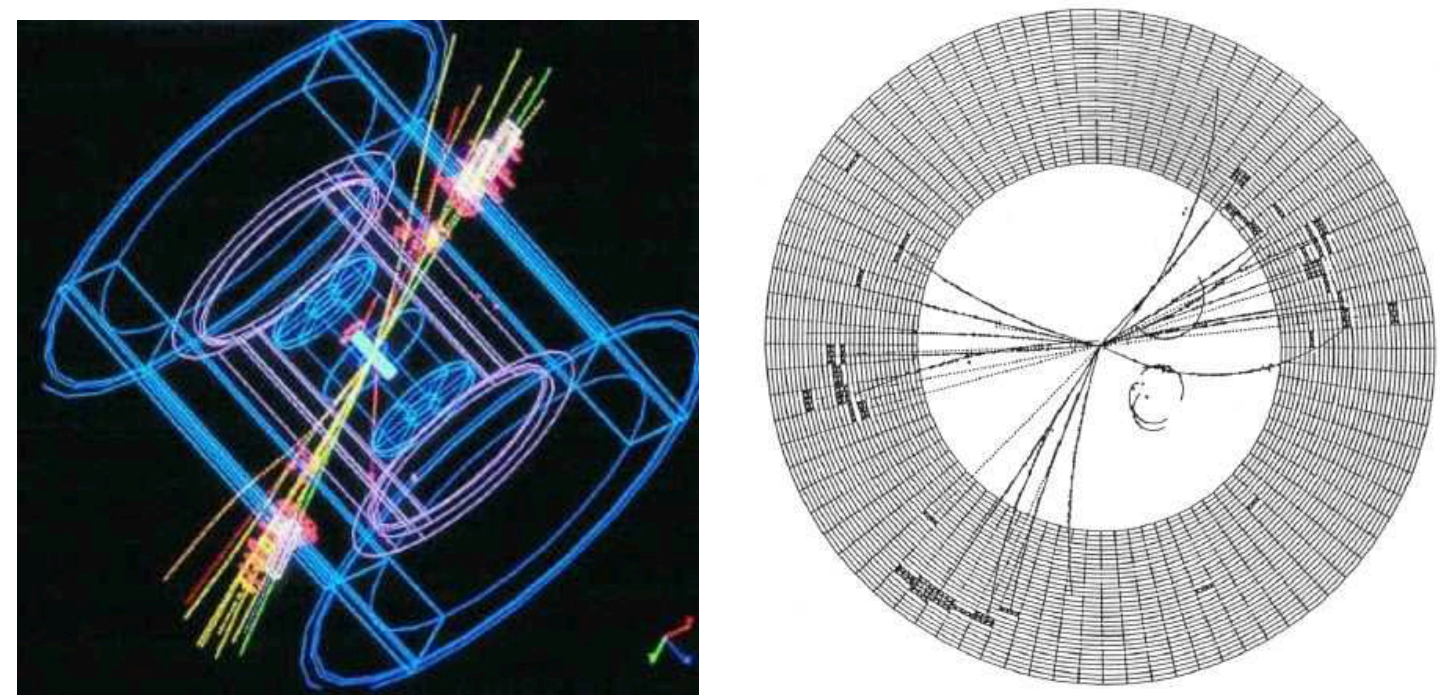

Fig. 14: Experimental pictures of 2- and 3-jet final states from $e^{+} e^{-}$collisions

of the final states, shown in fig. 12, corresponds to the many particles that emerge from the collinear emissions of many gluons from each quark. The dynamics of these emissions leads these particles to grossly follow the direction of the primary quark, and the emergent bundle, the jet, inherits the kinematics of the initial quark. This is shown in the left image of fig. 14. Three-jet events, shown in the right image of the figure, arise from the $O\left(\alpha_{s}\right)$ corrections to the tree-level process, namely to diagrams such as those shown in fig. 15.
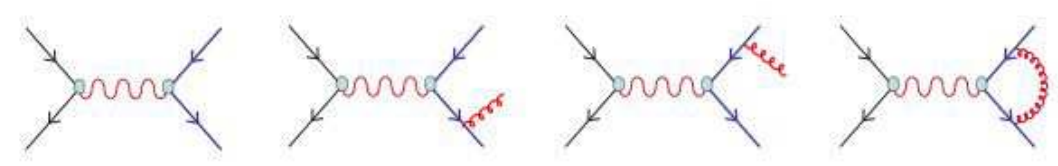

Fig. 15: $O\left(\alpha_{s}\right)$ corrections to the tree-level $e^{+} e^{-} \rightarrow q \bar{q}$ process

An important additional result of this 'pre-confining' evolution, is that the memory of where the local colour-neutral clusters came from is totally lost. So we expect the properties of hadronization to be 
universal: a model that describes hadronization at a given energy will work equally well at some other energy. Furthermore, so much time has passed since the original $q \bar{q}$ creation that the hadronization phase cannot significantly affect the total hadron production rate. Perturbative corrections due to the emission of the first hard partons should be calculable in PT, providing a finite, meaningful cross-section.

The nature of non-perturbative corrections to this picture can be explored. One can prove, for example, that the leading correction to the total rate $R_{e^{+} e^{-}}$is of order $F / s^{2}$, where $F \propto\left\langle 0\left|\alpha_{s} F_{\mu \nu}^{a} F^{\mu \nu a}\right| 0\right\rangle$ is the so-called gluon condensate. Since $F \sim \mathcal{O}\left(1 \mathrm{GeV}^{4}\right)$, these NP corrections are usually very small. For example, they are of $\mathcal{O}\left(10^{-8}\right)$ at the $Z^{0}$ peak. Corrections scaling like $\Lambda^{2} / s$ or $\Lambda / \sqrt{s}$ can nevertheless appear in other less inclusive quantities, such as event shapes or fragmentation functions.

We now come back to the perturbative evolution, and shall devote the first part of this lecture to justifying the picture given above.

\subsection{Soft gluon emission}

Emission of soft gluons plays a fundamental rôle in the evolution of the final state [7,13]. Soft gluons are emitted with large probability, since the emission spectrum behaves like $\mathrm{d} E / E$, typical of bremsstrahlung as familiar in QED. They provide the seed for the bulk of the final-state multiplicity of hadrons. The study of soft-gluon emission is simplified by the simplicity of their couplings. Being soft (i.e., long wavelength) they are insensitive to the details of the very-short-distance dynamics: they cannot distinguish features of the interactions which take place on time scales shorter than their wavelength. They are also insensitive to the spin of the partons: the only feature they are sensitive to is the colour charge. To prove this let us consider soft-gluon emission in the $q \bar{q}$ decay of an off-shell photon:

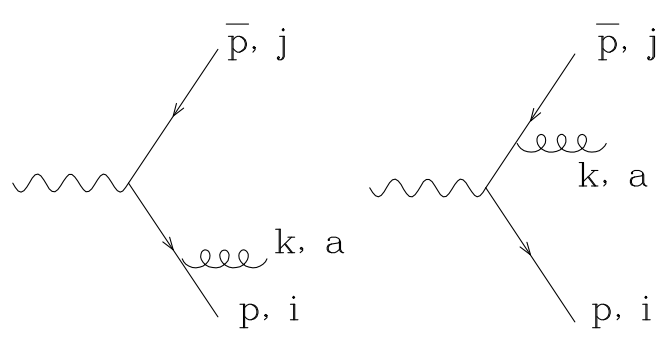

$$
\begin{aligned}
A_{\text {soft }} & =\bar{u}(p) \epsilon(k)(i g) \frac{-i}{\not p+\not k} \Gamma^{\mu} v(\bar{p}) \lambda_{i j}^{a}+\bar{u}(p) \Gamma^{\mu} \frac{i}{\not p+\not k}(i g) \epsilon(k) v(\bar{p}) \lambda_{i j}^{a} \\
& =\left[\frac{g}{2 p \cdot k} \bar{u}(p) \epsilon(k)(\not p+\not k) \Gamma^{\mu} v(\bar{p})-\frac{g}{2 \bar{p} \cdot k} \bar{u}(p) \Gamma^{\mu}(\not p+\not k) \epsilon(k) v(\bar{p})\right] \lambda_{i j}^{a} .
\end{aligned}
$$

I used the generic symbol $\Gamma_{\mu}$ to describe the interaction vertex with the photon to stress the fact that the following manipulations are independent of the specific form of $\Gamma_{\mu}$. In particular, $\Gamma_{\mu}$ can represent an arbitrarily complicated vertex form factor. Neglecting the factors of $\not k$ in the numerators (since $k \ll p, \bar{p}$, by definition of soft) and using the Dirac equations, we get:

$$
A_{\text {soft }}=g \lambda_{i j}^{a}\left(\frac{p \cdot \epsilon}{p \cdot k}-\frac{\bar{p} \epsilon}{\bar{p} \cdot k}\right) A_{\text {Born }}
$$


We then conclude that soft-gluon emission factorizes into the product of an emission factor, times the Born-level amplitude. From this exercise, one can extract general Feynman rules for soft-gluon emission:

a, $\mu$

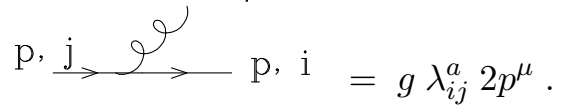

Exercise: Derive the $g \rightarrow g g$ soft-emission rules:

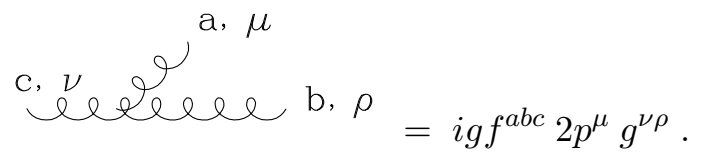

Example: Consider the 'decay' of a virtual gluon into a quark pair. One more diagram should be added to those considered in the case of the electroweak decay. The fact that the quark pair is no longer in a colour-singlet state makes things a bit more interesting:
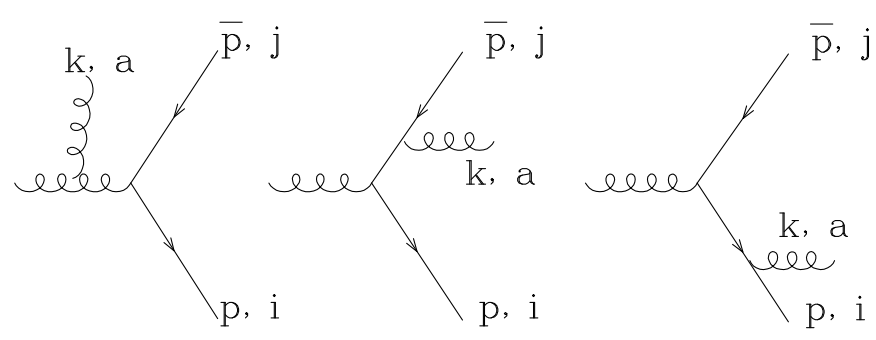

$$
\begin{aligned}
& \stackrel{k \rightarrow 0}{=}\left[i g f^{a b c} \lambda_{i j}^{c}\left(\frac{Q \epsilon}{Q k}\right)+g\left(\lambda^{b} \lambda^{a}\right)_{i j}\left(\frac{p \epsilon}{p k}\right)-g\left(\lambda^{a} \lambda^{b}\right)_{i j}\left(\frac{\bar{p} \epsilon}{p k}\right)\right] A_{\text {Born }} \\
& =g\left(\lambda^{a} \lambda^{b}\right)_{i j}\left[\frac{Q \epsilon}{Q k}-\frac{\bar{p} \epsilon}{p k}\right]+g\left(\lambda^{b} \lambda^{a}\right)_{i j}\left[\frac{p \epsilon}{p k}-\frac{Q \epsilon}{Q k}\right] .
\end{aligned}
$$

The two factors correspond to the two possible ways colour can flow in this process:

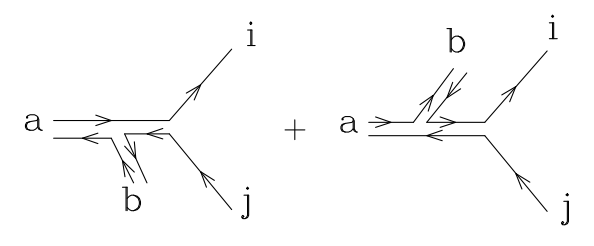

The basis for this representation of the colour flow is the following diagram which makes explicit the relation between the colours of the quark, antiquark, and gluon entering a QCD vertex:

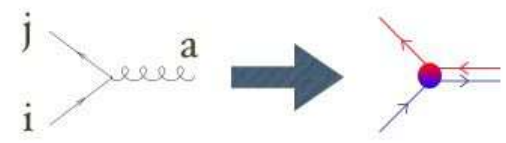


We can therefore represent the gluon as a double line, one line carrying the colour inherited from the quark, the other carrying the anticolour inherited from the antiquark. In the first diagram in (38) the antiquark (colour label $j$ ) is colour connected to the soft gluon (colour label $b$ ), and the quark (colour label $i$ ) is connected to the decaying gluon (colour label $a$ ). In the second case, the order is reversed. The two emission factors correspond to the emission of the soft gluon from the antiquark, and from the quark line, respectively. When squaring the total amplitude, and summing over initial and final-state colours, the interference between the two pieces is suppressed by $1 / N^{2}$ relative to the individual squares:

$$
\begin{aligned}
& \sum_{a, b, i, j}\left|\left(\lambda^{a} \lambda^{b}\right)_{i j}\right|^{2}=\sum_{a, b} \operatorname{tr}\left(\lambda^{a} \lambda^{b} \lambda^{b} \lambda^{a}\right)=\frac{N^{2}-1}{2} C_{F}=\mathcal{O}\left(N^{3}\right) . \\
& \sum_{a, b, i, j}\left(\lambda^{a} \lambda^{b}\right)_{i j}\left[\left(\lambda^{b} \lambda^{a}\right)_{i j}\right]^{*}=\sum_{a, b} \operatorname{tr}\left(\lambda^{a} \lambda^{b} \lambda^{a} \lambda^{b}\right)=\frac{N^{2}-1}{2} \underbrace{\left(C_{F}-\frac{C_{A}}{2}\right)}_{-\frac{1}{2 N}}=\mathcal{O}(N) .
\end{aligned}
$$

As a result, the emission of a soft gluon can be described, to the leading order in $1 / N^{2}$, as the incoherent sum of the emission from the two colour currents.

\subsection{Angular ordering for soft-gluon emission}

The results presented above have important consequences for the perturbative evolution of the quarks. A key property of the soft-gluon emission is the so-called angular ordering. This phenomenon consists in the continuous reduction of the opening angle at which successive soft gluons are emitted by the evolving quark. As a result, this radiation is confined within smaller and smaller cones around the quark direction, and the final state will look like a collimated jet of partons. In addition, the structure of the colour flow during the jet evolution forces the $q \bar{q}$ pairs which are in a colour-singlet state to be close in phase-space, thereby achieving the pre-confinement of colour-singlet clusters alluded to at the beginning of this section.

Let us start first by proving the property of colour ordering. Consider the $q \bar{q}$ pair produced by the decay of a rapidly moving virtual photon. The amplitude for the emission of a soft gluon was given in Eq. (33). Squaring, summing over colours, and including the gluon phase-space we get the following result:

$$
\begin{aligned}
d \sigma_{g} & =\sum\left|A_{\text {soft }}\right|^{2} \frac{d^{3} k}{(2 \pi)^{3} 2 k^{0}} \sum\left|A_{0}\right|^{2} \frac{-2 p^{\mu} \bar{p}^{\nu}}{(p k)(\bar{p} k)} g^{2} \sum \epsilon_{\mu} \epsilon_{\nu}^{*} \frac{d^{3} k}{(2 \pi)^{3} 2 k^{0}} \\
& =d \sigma_{0} \frac{2(p \bar{p})}{(p k)(\bar{p} k)} g^{2} C_{f}\left(\frac{d \phi}{2 \pi}\right) \frac{k^{0} d k^{0}}{8 \pi^{2}} d \cos \theta \\
& =d \sigma_{0} \frac{\alpha_{s} C_{F}}{\pi} \frac{d k^{0}}{k^{0}} \frac{d \phi}{2 \pi} \frac{1-\cos \theta_{i j}}{\left(1-\cos \theta_{i k}\right)\left(1-\cos \theta_{j k}\right)} d \cos \theta
\end{aligned}
$$

where $\theta_{\alpha \beta}=\theta_{\alpha}-\theta_{\beta}$, and $i, j, k$ refer to the $q, \bar{q}$ and gluon directions, respectively. We can write the following identity:

$$
\begin{aligned}
& \frac{1-\cos \theta_{i j}}{\left(1-\cos \theta_{i k}\right)\left(1-\cos \theta_{j k}\right)}= \\
& \frac{1}{2}\left[\frac{\cos \theta_{j k}-\cos \theta_{i j}}{\left(1-\cos \theta_{i k}\right)\left(1-\cos \theta_{j k}\right)}+\frac{1}{1-\cos \theta_{i k}}\right]+\frac{1}{2}[i \leftrightarrow j] \equiv W_{(i)}+W_{(j)} .
\end{aligned}
$$

We would like to interpret the two functions $W_{(i)}$ and $W_{(j)}$ as radiation probabilities from the quark and antiquark lines. Each of them is in fact only singular in the limit of gluon emission parallel to the respective quark:

$$
W_{(i)} \rightarrow \text { finite if } k \| j\left(\cos \theta_{j k} \rightarrow 1\right)
$$




$$
W_{(j)} \rightarrow \text { finite if } k \| i\left(\cos \theta_{i k} \rightarrow 1\right) .
$$

The intepretation as probabilities is however limited by the fact that neither $W_{(i)}$ nor $W_{(j)}$ are positive definite. However, you can easily prove that

$$
\int \frac{d \phi}{2 \pi} W_{(i)}=\left\{\begin{array}{cc}
\frac{1}{1-\cos \theta_{i k}} & \text { if } \theta_{i k}<\theta_{i j} \\
0 & \text { otherwise }
\end{array}\right.
$$

where the integral is the azimuthal average around the $q$ direction. A similar result holds for $W_{(j)}$ :

$$
\int \frac{d \phi}{2 \pi} W_{(j)}=\left\{\begin{array}{cc}
\frac{1}{1-\cos \theta_{j k}} & \text { if } \theta_{j k}<\theta_{i j} \\
0 & \text { otherwise }
\end{array}\right.
$$

As a result, the emission of soft gluons outside the two cones obtained by rotating the antiquark direction around the quark's, and vice versa, averages to 0 . Inside the two cones, one can consider the radiation from the emitters as being uncorrelated. In other words, the two colour lines defined by the quark and antiquark currents act as independent emitters, and the quantum coherence (i.e. the effects of interference between the two graphs contributing to the gluon-emission amplitude) is accounted for by constraining the emission to take place within those fixed cones.
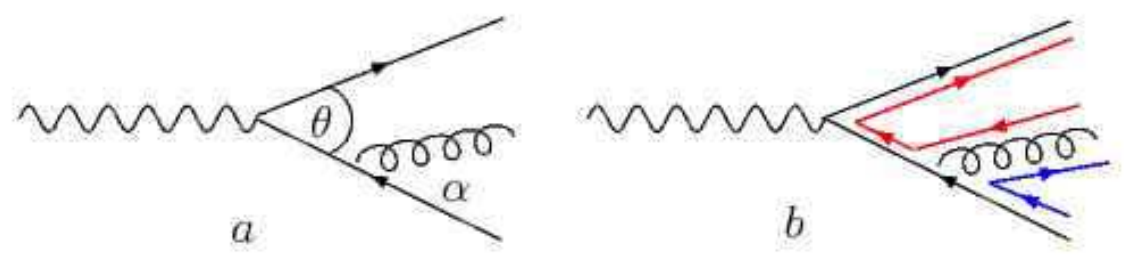

Fig. 16: Radiation off $q \bar{q}$ pair produced by an off-shell photon

A simple derivation of angular ordering, which more directly exhibits its physical origin, can be obtained as follows. Consider Fig. 16(a), which shows a Feynman diagram for the emission of a gluon from a quark line. The quark momentum is denoted by $l$ and the gluon momentum by $k, \theta$ is the opening angle between the quark and antiquark, and $\alpha$ is the angle between the nearest quark and the emitted gluon. We shall work in the double-log enhanced soft $k^{0}<<l^{0}$ and collinear $\alpha<<1$ region. The internal quark propagator $p=(l+k)$ is off-shell, setting the time scale for the gluon emission:

$$
\Delta t \simeq \frac{1}{\Delta E}=\frac{l^{0}}{(k+l)^{2}} \quad \rightarrow \quad \Delta t \simeq \frac{1}{k^{0} \alpha^{2}} .
$$

In order to resolve the quarks, the transverse wavelength of the gluon $\lambda_{\perp}=1 / E_{\perp}$ must be smaller than the separation between the quarks $b(t) \simeq \theta \Delta t$, giving the constraint $1 /\left(\alpha k^{0}\right)<\theta \Delta t$. Using the results of Eq. 48 for $\Delta t$, we arrive at the angular ordering constraint $\alpha<\theta$. Gluon emissions at an angle smaller than $\theta$ can resolve the two individual colour quarks and are allowed; emissions at greater angles do not see the colour charge and are therefore suppressed. In processes involving more partons, the angle $\theta$ is defined not by the nearest parton, but by the colour connected parton (e.g. the parton that forms a colour singlet with the emitting parton). Figure 16(b) shows the colour connections for the $q \bar{q}$ event after the gluon is emitted. Colour lines begin on quarks and end on antiquarks. Because gluons are colour octets, they contain the beginning of one line and the end of another, as we showed in (38).

If one repeats now the exercise for emission of one additional gluon, one will find the same angular constraint, but this time applied to the colour lines defined by the previously established antenna. As 
shown in the previous subsection, the $q \bar{q} g$ state can be decomposed at the leading order in $1 / N$ into two independent emitters, one given by the colour line flowing from the gluon to the quark, the other given by the colour line flowing from the antiquark to the gluon. So the emission of the additional gluon will be constrained to take place either within the cone formed by the quark and the gluon, or within the cone formed by the gluon and the antiquark. Either way, the emission angle will be smaller than the angle of the first gluon emission. This leads to the concept of angular ordering, with successive emission of soft gluons taking place within cones which get smaller and smaller, as in Fig. 17

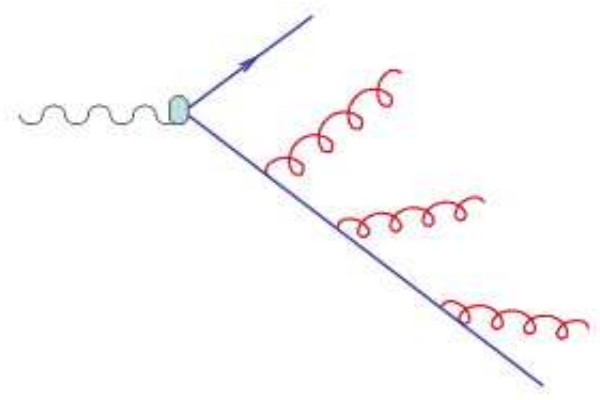

Fig. 17: Collimation of soft gluon emission during the jet evolution

The fact that colour always flows directly from the emitting parton to the emitted one, the collimation of the jet, and the softening of the radiation emitted at later stages, ensure that partons forming a colour-singlet cluster are close in phase-space. As a result, hadronization (the non-perturbative process that will bind together colour-singlet parton pairs) takes place locally inside the jet and is not a longdistance phenomenon connecting partons far away in the evolution tree: only pairs of nearby partons are involved. In particular, there is no direct link between the precise nature of the hard process and the hadronization. These two phases are totally decoupled and, as in the case of the partonic densities, one can infer that hadronization factorizes from the hard process and can be described in a universal (i.e. hard-process independent) fashion. The inclusive properties of jets (e.g. the particle multiplicity, jet mass, jet broadening) are independent of the hadronization model, up to corrections of order $(\Lambda / \sqrt{s})^{n}$ (for some integer power $n$, which depends on the observable), with $\Lambda \lesssim 1 \mathrm{GeV}$.

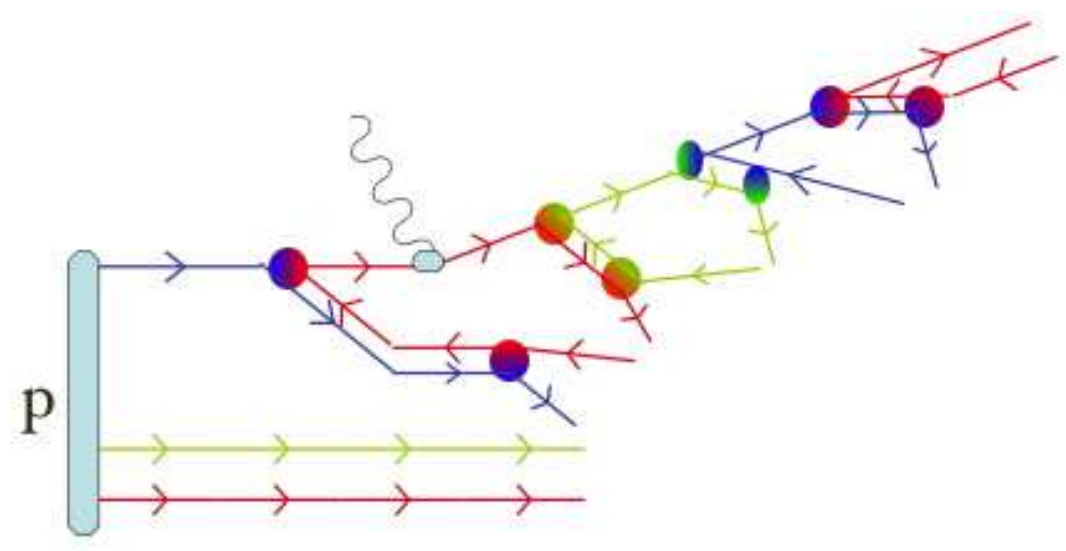

Fig. 18: The colour flow diagram for a DIS event

The final picture, in the case of a DIS event, appears therefore as in Fig. 18. After being deflected by the photon, the struck quark emits the first gluon that takes away the quark colour and passes on its own anticolour to the escaping quark. This gluon is therefore colour-connected with the last gluon emitted 
before the hard interaction. As the final-state quark continues its evolution, more and more gluons are emitted, each time leaving their colour behind and transmitting their anticolour to the emerging quark. Angular ordering forces all these gluons to be close in phase-space, until the evolution is stopped once the virtuality of the quark becomes of the order of the strong-interaction scale. The colour of the quark is left behind, and when hadronization takes over it is only the nearby colour-connected gluons which are transformed, with a phenomenological model, in hadrons. This mechanism for the transfer of colour across subsequent gluon emissions is similar to what happens when we place a charge near the surface of a dielectric medium. This will become polarized, and a charge will appear on the medium opposite end. The appearance of the charge is the result of a sequence of local charge shifts, whereby neighbouring atoms get polarized, as in Fig. 19.
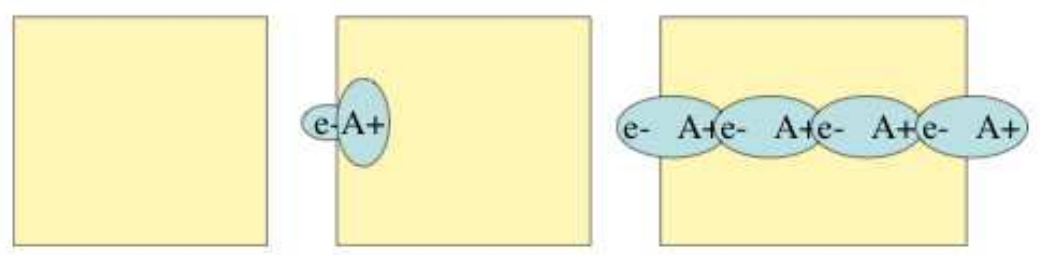

Fig. 19: Charge transfer in a dieletric medium, via a sequence of local polarizations

\subsection{Hadronization}

The application of perturbation theory to the evolution of a jet, with the sequential emission of partons, governed by QCD splitting probabilities and angular ordering to enforce quantum mechanical quantum coherence, will stop once the scale of the emissions reaches values in the range of $1 \mathrm{GeV}$. This is called the infrared cutoff. The are two reasons why we need to stop the emission of gluons at this scale. To start with, we cannot control with perturbation theory the domain below this scale, where the strong coupling constant $\alpha_{s}$ becomes very large. Furthermore, we know that the number of physical particles that can be produced inside a jet must be finite, since the lightest object we can produce is a pion, and energy conservation sets a limit to how many pions can be created. This is different from what happens in a QED cascade, where the evolution of an accelerated charge can lead to the emission of an arbitrary number of photons. This is possible because the photon is massless, and can have arbitrarily small energy. The gluons of a QCD cascade, on the contrary, must have enough energy to create pions.

When the perturbative evolution of the jet terminates, we are left with some number of gluons. As shown in the previous subsection, and displayed in fig. 18, these gluons are pairwise colour connected. As two colour-connected gluons travel away from each other, a constant force pulls them together. Phenomenological models (see Ref. [7] for a more complete review) are then used to describe how this force determines the evolution of the system from this point on. What I shall describe here is the so-called cluster model [17], but the main qualitative features are shared by other alternatives, such as the Lund string approach [18].

Most of the hadrons emerging from the evolution of a jet are known to be made of quarks; glueballs, i.e. hadrons made of bound gluons, are expected to exist, but their production is greatly suppressed compared to that of quark-made particles. For this reason, the first step in the description of hadronization is to assume that the force among gluons will rip them apart into a $q \bar{q}$ pair, and that these quarks will act as seeds for the hadron production. The break-up into quarks is not parametrized using the DGLAP $g \rightarrow q \bar{q}$ splitting function, since we are deaing here with a non-perturbative transition. One typically employs therefore a pure phase-space 'decay' of the gluon into the $q \bar{q}$ pair, introducing as phenomenological parameters the relative probabilities of selecting the various active flavours (up, down, strange, etc.). The quark $q_{i}$ from one gluon ( $i$ representing the flavour) then forms a colour-singlet pair with the 
antiquark $\bar{q}_{j}$ emerging from the break-up of the neighboring gluon. This colour-singlet $q_{i} \bar{q}_{j}$ pair cannot, however, directly form a hadron, since in general the quarks will still be moving apart, and the invariant mass of the pair will not coincide with the mass of an existing physical state. As they separate subject to

Primary Light Clusters

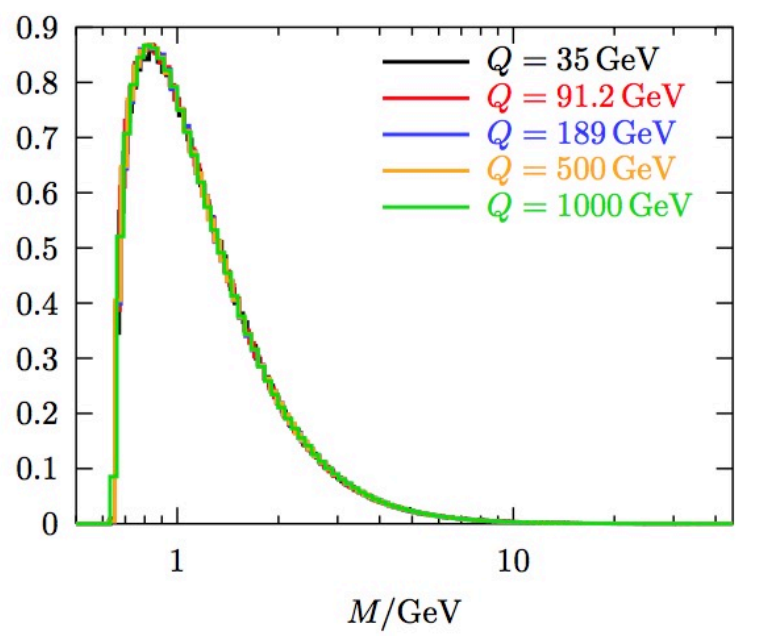

Fig. 20: Invariant mass distribution of clusters of colour-singlet quarks after non-perturbative gluon splitting. The spectra for final states corresponding to different centre-of-mass energies are normalized to the same area, displaying the energy independence of the shapes

a constant force, however, their kinetic energy turns into a linearly-rising potential energy. The potential energy accumulated in the system will be able to convert into a new quark-antiquark pair, $q_{k} \bar{q}_{k}$ once its value exceeds the relevant mass threshold. We are now left with two colour-singlet pairs, $q_{i} \bar{q}_{k}$ and $q_{k} \bar{q}_{j}$. One can force the kinematics of the $q_{k} \bar{q}_{k}$ pair to allow for both $q_{i} \bar{q}_{k}$ and $q_{k} \bar{q}_{j}$ invariant masses to coincide with some resonance with the proper flavour. The residual energy of the system is then assumed to be entirely kinetic, and the two resonances fly away free. Once again, one can associate phenomenological parameters to the probabilities of selecting flavours $k$ of a given type. Since the pair of flavour indices $i k$ does not specify uniquely a hadron (e.g. a $u \bar{d}$ system could by a $\pi^{+}$, a $\rho^{+}$, as well as many other objects), the model has a further set of rules and/or parameters to select the precise flavour type. For example, a phenomenologically successfull description of the $\pi / \rho$ ratio is obtained by simply assuming a production rate proportional to the number of spin states (one for the scalar pion, three for the vector rho) and to a Boltzmann factor $\exp (-M / T)$, where $M$ is the resonance mass and $T$ is a universal parameter, to be fit from data. Furthermore, one can introduce the possibility of converting the potential energy into a diquark-antidiquark pair, namely $\left(q_{k} q_{\ell}\right)\left(\bar{q}_{k} \bar{q}_{\ell}\right)$. The resulting hadrons, $q_{i} q_{k} q_{\ell}$ and $\bar{q}_{j} \bar{q}_{k} \bar{q}_{\ell}$ will be a baryon-antibaryon pair.

The measurement of hadron multiplicities from $Z^{0}$ decays is used to tune the few phenomenological parameters of the model, and these parameters can be used to describe hadronization at different energies and in different high-energy hadron-production processes. The internal consistency of this assumption is supported by fig. 20, which shows the invariant mass distribution of clusters of colour-singlet quarks, after the non-perturbative gluon splitting, for $e^{+} e^{-}$collisions at different center-of-mass energies. All curves are normalized to 1 , and they all overlap very accurately. This confirms the validity of the implementation of factorization in the Monte Carlo: higher initial energies provide more room for the perturbative evolution, leading to more splitting and more emitted radiation; but the structure and distribution of colour-singlet clusters at the end of evolution is independent of the initial energy, and the same model of hadronization can be applied. 
Table 1: Average particle multiplicities per event in $e^{+} e^{-}$collisions at $91.2 \mathrm{GeV}$. Experimental data were measured by the following collaborations at LEP and at SLC: ALEPH(A), DELPHI(D), L3(L), OPAL(O), MARK2(M) and $\operatorname{SLD}(\mathrm{S})$. The theoretical predictions in the last three columns, taken from Ref. [19], correspond to various implementations of the cluster hadronization model (see Ref. [19] for details). The $*$ indicates a prediction that differs from the measured value by more than three standard deviations.

\begin{tabular}{|c|c|c|c|c|c|}
\hline Particle & Experiment & Measured & Old Model & Herwig++ & Fortran \\
\hline All Charged & $\mathrm{M}, \mathrm{A}, \mathrm{D}, \mathrm{L}, \mathrm{O}$ & $20.924 \pm 0.117$ & $20.22^{*}$ & 20.814 & $20.532^{*}$ \\
\hline$\gamma$ & $\mathrm{A}, \mathrm{O}$ & $21.27 \pm 0.6$ & 23.03 & 22.67 & 20.74 \\
\hline$\pi^{0}$ & A,D,L,O & $9.59 \pm 0.33$ & 10.27 & 10.08 & 9.88 \\
\hline$\rho(770)^{0}$ & A,D & $1.295 \pm 0.125$ & 1.235 & 1.316 & 1.07 \\
\hline$\pi^{ \pm}$ & $\mathrm{A}, \mathrm{O}$ & $17.04 \pm 0.25$ & 16.30 & 16.95 & 16.74 \\
\hline$\rho(770)^{ \pm}$ & $\mathrm{O}$ & $2.4 \pm 0.43$ & 1.99 & 2.14 & 2.06 \\
\hline$\eta$ & $\mathrm{A}, \mathrm{L}, \mathrm{O}$ & $0.956 \pm 0.049$ & 0.886 & 0.893 & $0.669^{*}$ \\
\hline$\omega(782)$ & $\mathrm{A}, \mathrm{L}, \mathrm{O}$ & $1.083 \pm 0.088$ & 0.859 & 0.916 & 1.044 \\
\hline$\eta^{\prime}(958)$ & $\mathrm{A}, \mathrm{L}, \mathrm{O}$ & $0.152 \pm 0.03$ & 0.13 & 0.136 & 0.106 \\
\hline$K^{0}$ & $\mathrm{~S}, \mathrm{~A}, \mathrm{D}, \mathrm{L}, \mathrm{O}$ & $2.027 \pm 0.025$ & $2.121^{*}$ & 2.062 & 2.026 \\
\hline$K^{*}(892)^{0}$ & $\mathrm{~A}, \mathrm{D}, \mathrm{O}$ & $0.761 \pm 0.032$ & 0.667 & 0.681 & $0.583^{*}$ \\
\hline$K^{*}(1430)^{0}$ & $\mathrm{D}, \mathrm{O}$ & $0.106 \pm 0.06$ & 0.065 & 0.079 & 0.072 \\
\hline$K^{ \pm}$ & $\mathrm{A}, \mathrm{D}, \mathrm{O}$ & $2.319 \pm 0.079$ & 2.335 & 2.286 & 2.250 \\
\hline$K^{*}(892)^{ \pm}$ & $\mathrm{A}, \mathrm{D}, \mathrm{O}$ & $0.731 \pm 0.058$ & 0.637 & 0.657 & 0.578 \\
\hline$\phi(1020)$ & $\mathrm{A}, \mathrm{D}, \mathrm{O}$ & $0.097 \pm 0.007$ & 0.107 & 0.114 & $0.134^{*}$ \\
\hline$p$ & $\mathrm{~A}, \mathrm{D}, \mathrm{O}$ & $0.991 \pm 0.054$ & 0.981 & 0.947 & 1.027 \\
\hline$\Delta^{++}$ & $\mathrm{D}, \mathrm{O}$ & $0.088 \pm 0.034$ & 0.185 & 0.092 & $0.209^{*}$ \\
\hline$\Sigma^{-}$ & $\mathrm{O}$ & $0.083 \pm 0.011$ & 0.063 & 0.071 & 0.071 \\
\hline$\Lambda$ & A,D,L,O & $0.373 \pm 0.008$ & $0.325^{*}$ & 0.384 & $0.347^{*}$ \\
\hline$\Sigma^{0}$ & $\mathrm{~A}, \mathrm{D}, \mathrm{O}$ & $0.074 \pm 0.009$ & 0.078 & 0.091 & 0.063 \\
\hline$\Sigma^{+}$ & $\mathrm{O}$ & $0.099 \pm 0.015$ & 0.067 & 0.077 & 0.088 \\
\hline$\Sigma(1385)^{ \pm}$ & $\mathrm{A}, \mathrm{D}, \mathrm{O}$ & $0.0471 \pm 0.0046$ & 0.057 & $0.0312^{*}$ & $0.061^{*}$ \\
\hline$\Xi^{-}$ & $\mathrm{A}, \mathrm{D}, \mathrm{O}$ & $0.0262 \pm 0.001$ & 0.024 & 0.0286 & 0.029 \\
\hline$\Xi(1530)^{0}$ & $\mathrm{~A}, \mathrm{D}, \mathrm{O}$ & $0.0058 \pm 0.001$ & $0.026^{*}$ & $0.0288^{*}$ & $0.009^{*}$ \\
\hline$\Omega^{-}$ & $\mathrm{A}, \mathrm{D}, \mathrm{O}$ & $0.00125 \pm 0.00024$ & 0.001 & 0.00144 & 0.0009 \\
\hline$f_{2}(1270)$ & $\mathrm{D}, \mathrm{L}, \mathrm{O}$ & $0.168 \pm 0.021$ & 0.113 & 0.150 & 0.173 \\
\hline$f_{2}^{\prime}(1525)$ & $\mathrm{D}$ & $0.02 \pm 0.008$ & 0.003 & 0.012 & 0.012 \\
\hline$D^{ \pm}$ & $\mathrm{A}, \mathrm{D}, \mathrm{O}$ & $0.184 \pm 0.018$ & $0.322^{*}$ & $0.319^{*}$ & $0.283^{*}$ \\
\hline$D^{*}(2010)^{ \pm}$ & $\mathrm{A}, \mathrm{D}, \mathrm{O}$ & $0.182 \pm 0.009$ & 0.168 & 0.180 & $0.151^{*}$ \\
\hline$D^{0}$ & $\mathrm{~A}, \mathrm{D}, \mathrm{O}$ & $0.473 \pm 0.026$ & $0.625^{*}$ & $0.570^{*}$ & 0.501 \\
\hline$D_{s}^{ \pm}$ & $\mathrm{A}, \mathrm{O}$ & $0.129 \pm 0.013$ & $0.218^{*}$ & $0.195^{*}$ & 0.127 \\
\hline$D_{s}^{* \pm}$ & $\mathrm{O}$ & $0.096 \pm 0.046$ & 0.082 & 0.066 & 0.043 \\
\hline$J / \Psi$ & $\mathrm{A}, \mathrm{D}, \mathrm{L}, \mathrm{O}$ & $0.00544 \pm 0.00029$ & 0.006 & $0.00361^{*}$ & $0.002^{*}$ \\
\hline$\Lambda_{c}^{+}$ & $\mathrm{D}, \mathrm{O}$ & $0.077 \pm 0.016$ & $0.006^{*}$ & $0.023^{*}$ & $0.001^{*}$ \\
\hline$\Psi^{\prime}(3685)$ & $\mathrm{D}, \mathrm{L}, \mathrm{O}$ & $0.00229 \pm 0.00041$ & $0.001^{*}$ & 0.00178 & $0.0008^{*}$ \\
\hline
\end{tabular}

An example of the quality of the fits to $Z^{0}$-decay data is given in table 1 , which is taken from [19]. There more details are given on the possible variants of cluster hadronization model and on the choice of parameters used in the fits. Overall, the agreement is excellent!

\section{Applications to hadronic collisions}

In hadronic collisions all phenomena are QCD related. The dynamics is more complex than in $e^{+} e^{-}$ or DIS, since both beam and target have a non-trivial partonic structure. As a result, calculations (and experimental analyses) are more complicated. QCD phenomenology is however much richer, and the higher energies available in hadronic collisions allow one to probe the structure of the proton and of its 
constituents at the smallest scales attainable in a laboratory, in addition to probing the large scales where new physics phenomena may appear.

Recent remarkable progress in thoeretical calculations, furthermore, has allowed to achieve nextto-leading-order (NLO) accuracy for all observables of interest, and a large majority of these can now be calculated also to next-to-next-to-leading order (NNLO). The overall precision in the theoretical predictions is therefore reaching now the level of accuracy that until recently was only imaginable for $e^{+} e^{-}$or $e p$ collisions.

The key ingredients for the calculation of production rates and distributions in hadronic collisions are

- the matrix elements for the hard, partonic process (e.g., $g g \rightarrow g g, g g \rightarrow b \bar{b}, q \bar{q}^{\prime} \rightarrow W, \ldots$ ),

- the hadronic parton densities, discussed in the previous lecture.

Then the production rate for a given final state $H$ is given by a factorization formula similar to the one used to describe DIS:

$$
d \sigma(p \bar{p} \rightarrow H+X)=\int d x_{1} d x_{2} \sum_{i, j} f_{i}\left(x_{1}, Q\right) f_{j}\left(x_{2} \cdot Q\right) d \hat{\sigma}(i j \rightarrow H) .
$$

where the parton density $f_{i}$ 's are evaluated at a scale $Q$ typical of the hard process under consideration. For example $Q \simeq M_{D Y}$ for production of a Drell-Yan pair, $Q \simeq E_{T}$ for high transverse-energy $\left(E_{T}\right)$ jets, $Q^{2} \simeq p_{T}^{2}+m_{Q}^{2}$ for high- $p_{T}$ heavy quarks, etc.

In this lecture we shall briefly explore two of the QCD phenomena currently studied in hadronic collisions: Drell-Yan, and inclusive jet production. More details can be found in Refs. [7, 8].

\subsection{QCD aspects of inclusive vector boson production}

The main feature of inclusive gauge boson production in hadronic collisions is that the leading-order (LO) amplitude, describing the elementary process $q \bar{q}^{\left({ }^{\prime}\right)} \rightarrow V(V=W, Z)$ is purely EW. The dynamics of strong interactions, at this order, only enters indirectly through the parton distribution functions (PDFs), which parameterize in a phenomenological way the quark and gluon content of the proton. At the large momentum scales typical of gauge boson production $\left(Q \sim M_{V}\right)$, higher-order perturbative QCD corrections to the inclusive production are proportional to $\alpha_{s}(Q)$ and are typically small, in the range of $10-20 \%$. They are known $[20,21]$ today to next-to-next-to-leading order (NNLO), including the description of the differential distributions of the boson and of its decay leptons [22-25], leaving theoretical uncertainties from higher-order QCD effects at the percent level. These results have been incorporated in full Monte Carlo calculations including the shower evolution, to give a complete description of the physical final states [26-28]. Next-to-leading-order (NLO) EW corrections are also known [29-32], and play an important role both for precision measurements, and in the production rate of dilepton pairs at large $p_{T}$ or with large mass, above the $\mathrm{TeV}$, where they can be larger than $10 \%$. Finally, progress towards a complete calculation of the mixed $\mathcal{O}\left(\alpha_{s} \alpha\right)$ corrections has been recently reported in Ref. [33].

When considering the first and second generation quarks that dominate the production of $W$ and $Z$ bosons, their weak couplings, including the CKM mixing parameters, are known experimentally with a precision better than a percent. This exceeds the accuracy of possible measurements in hadronic collisions, indicating that such measurements could not be possibly affected, at this level of precision, by the presence of new physics phenomena. They therefore provide an excellent ground to probe to percent precision the effects of higher-order QCD corrections and of PDFs [34]. To be more explicit, consider the leading-order (LO) cross section given by eq. 49 . In the case of $W$ production (a similar result holds for the $Z$ ), the LO partonic cross section is given by:

$$
\hat{\sigma}\left(q_{i} \bar{q}_{j} \rightarrow W\right)=\pi \frac{\sqrt{2}}{3}\left|V_{i j}\right|^{2} G_{F} M_{W}^{2} \delta\left(\hat{s}-M_{W}^{2}\right)=A_{i j} M_{W}^{2} \delta\left(\hat{s}-M_{W}^{2}\right)
$$


Here $\hat{s}=x_{1} x_{2} S$ is the partonic centre-of-mass energy squared, and $V_{i j}$ is the element of the CabibboKobayashi-Maskawa (CKM) matrix.

Written in terms of $\tau=x_{1} x_{2}$ and of the rapidity $y=\log \left[\left(E_{W}+p_{W}^{z}\right) /\left(E_{W}-p_{W}^{z}\right)\right]^{1 / 2} \equiv$ $\log \left(x_{1} / x_{2}\right)^{1 / 2}$, the differential and total cross sections are given by:

$$
\begin{aligned}
\frac{d \sigma_{W}}{d y} & =\sum_{i, j} \frac{\pi A_{i j}}{M_{W}^{2}} \tau f_{i}\left(x_{1}\right) f_{j}\left(x_{2}\right), \quad x_{1,2}=\sqrt{\tau} e^{ \pm y} \\
\sigma_{W} & =\sum_{i, j} \frac{\pi A_{i j}}{M_{W}^{2}} \tau \int_{\tau}^{1} \frac{d x}{x} f_{i}(x) f_{j}\left(\frac{\tau}{x}\right) \equiv \sum_{i, j} \frac{\pi A_{i j}}{M_{W}^{2}} \tau L_{i j}(\tau)
\end{aligned}
$$

where the function $L_{i j}(\tau)$ is usually called partonic luminosity. In the case of $u \bar{d}$ collisions, $\frac{\pi A_{i j}}{M_{W}^{2}} \sim$ $6.5 \mathrm{nb}$. It is interesting to study the partonic luminosity as a function of the hadronic centre-of-mass energy. This can be done by taking a simple approximation for the parton densities. Using the approximate behaviour $f_{i}(x) \sim 1 / x^{1+\delta}$, with $\delta<1$, one easily obtains:

$$
L(\tau)=\frac{1}{\tau^{1+\delta}} \log \left(\frac{1}{\tau}\right) \quad \text { and } \quad \sigma_{W} \propto\left(\frac{S}{M_{W}^{2}}\right)^{\delta} \log \left(\frac{S}{M_{W}^{2}}\right) .
$$

The gauge boson production cross section grows therefore at least logarithmically with the hadronic centre-of-mass energy.

NNLO $\mathrm{W}^{+}$and $\mathrm{W}^{-}$cross sections at the LHC $(\sqrt{s}=7 \mathrm{TeV})$

NNLO $W$ and $Z$ cross sections at the LHC $(\sqrt{s}=7 \mathrm{TeV})$
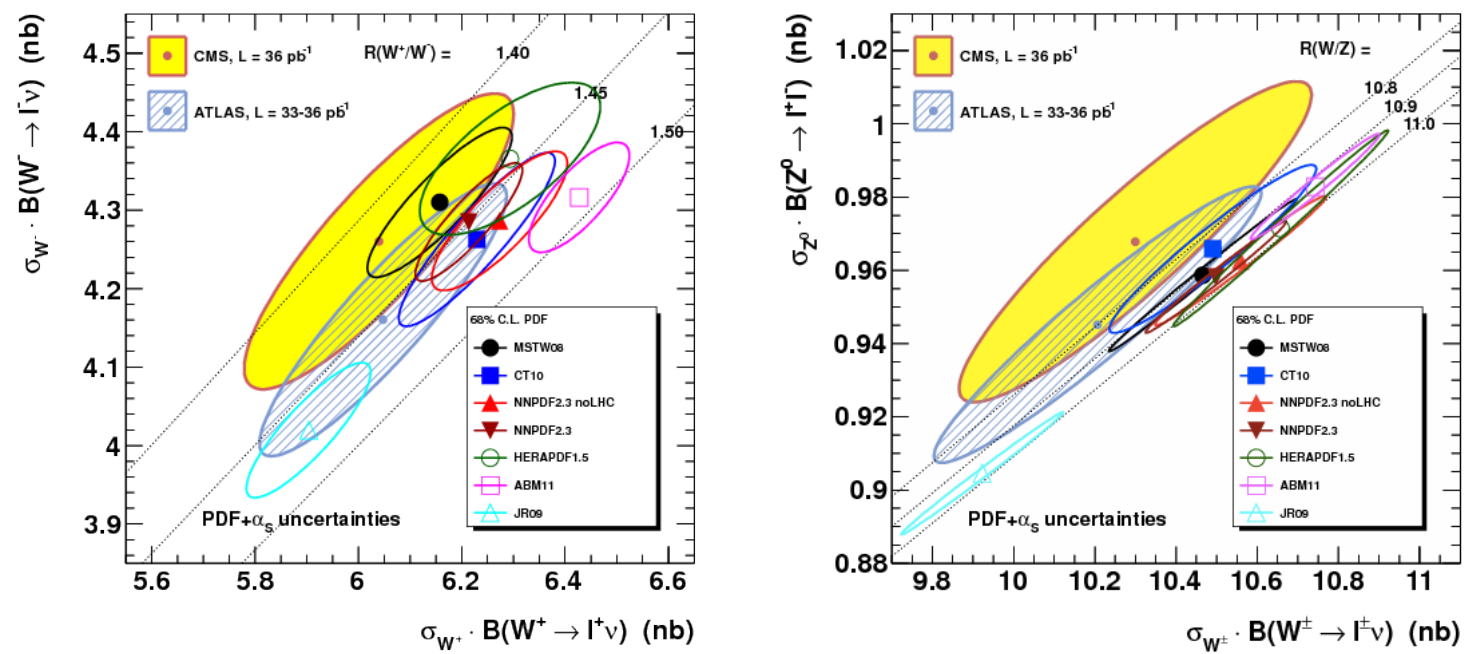

Fig. 21: $W$ and $Z$ boson cross sections in $p p$ collisions at $\sqrt{S}=7 \mathrm{TeV}$ : ATLAS [35] and CMS [36] data, compared to NNLO predictions for various PDF sets [34].

\subsubsection{Rapidity spectrum of $W$ and $Z$ bosons and $W$ charge asymmetries}

The features of the momentum distribution of vector bosons along the beam direction $(z)$ are mostly controlled by properties of the parton PDFs. In particular, in the case of $W$ bosons, the differences between the PDFs of up- and down-type quarks and antiquarks lead to interesting production asymmetries. Since the measurement of asymmetries is typically very accurate, due to the cancellation of many experimental and theoretical uncertainties, these play a fundamental role in the precision determination of quark and antiquark PDFs. Furthermore, the production asymmetries are modulated by the parity violation of the 
vector boson couplings, leading to further handles for the discrimination of quark and antiquark densities, and inducing a sensitivity to the weak mixing angle $\sin ^{2} \theta_{\text {eff }}^{\text {lept }}$, which controls the vector and axial components of $Z$ boson interactions.

For $p \bar{p}$ collisions, and assuming for simplicity the dominance of $u$ and $d$ quarks, we have:

$$
\begin{aligned}
\frac{d \sigma_{W^{+}}}{d y} & \propto f_{u}^{p}\left(x_{1}\right) f_{\bar{d}}^{\bar{p}}\left(x_{2}\right)+f_{\bar{d}}^{p}\left(x_{1}\right) f_{u}^{\bar{p}}\left(x_{2}\right) \\
\frac{d \sigma_{W^{-}}}{d y} & \propto f_{\bar{u}}^{p}\left(x_{1}\right) f_{d}^{\bar{p}}\left(x_{2}\right)+f_{d}^{p}\left(x_{1}\right) f_{\bar{u}}^{\bar{p}}\left(x_{2}\right)
\end{aligned}
$$

We can then construct the following charge asymmetry (using $f_{q}^{\bar{p}}=f_{\bar{q}}^{p}$ and assuming the dominance of the quark densities over the antiquark ones, which is valid in the kinematical region of interest for $W$ production at the Tevatron):

$$
A(y)=-A(-y)=\frac{\frac{d \sigma_{W^{+}}}{d y}-\frac{d \sigma_{W^{-}}}{d y}}{\frac{d \sigma_{W^{+}}}{d y}+\frac{d \sigma_{W^{-}}}{d y}} \sim \frac{f_{u}^{p}\left(x_{1}\right) f_{d}^{p}\left(x_{2}\right)-f_{d}^{p}\left(x_{1}\right) f_{u}^{p}\left(x_{2}\right)}{f_{u}^{p}\left(x_{1}\right) f_{d}^{p}\left(x_{2}\right)+f_{d}^{p}\left(x_{1}\right) f_{u}^{p}\left(x_{2}\right)}
$$

Setting $f_{u}(x)=f_{d}(x) R(x)$ we then get:

$$
A(y) \sim \frac{R\left(x_{1}\right)-R\left(x_{2}\right)}{R\left(x_{1}\right)+R\left(x_{2}\right)},
$$

which gives an explicit relation between asymmetry and the functional dependence of the $u(x) / d(x)$ ratio. This ratio is close to 1 at small $x$, where the quark distributions arise mostly from sea quarks, and it increases at larger $x$, where the valence contribution dominates. At positive $y$, where $x_{1}>x_{2}$, we therefore expect a positive asymmetry. This is confirmed in the left plot of Fig. 22, showing the asymmetry measured at the Tevatron by the CDF experiment [38], and compared to the NNLO QCD prediction $[24,32,39,40]$ and an estimate of the PDF uncertainty. When measuring the charged lepton
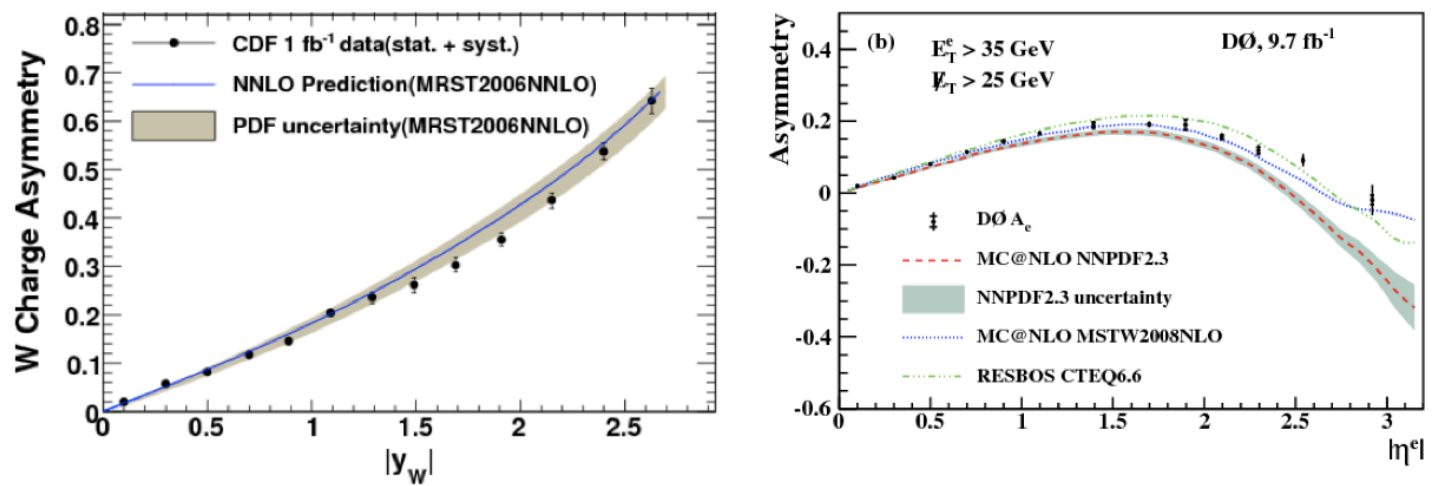

Fig. 22: Production [38] (left) and leptonic [41] (right) charge asymmetries of $W$ bosons in $p \bar{p}$ collisions at the Tevatron, $\sqrt{S}=1.96 \mathrm{TeV}$.

from $W$ decay, the $W$ production asymmetry is however modulated by the $W$ decay asymmetry caused by parity violation. The squared amplitude for the process $f_{1} \bar{f}_{2} \rightarrow W \rightarrow f_{3} \bar{f}_{4}$ is proportional to $\left(p_{1} \cdot p_{4}\right)\left(p_{2} \cdot p_{3}\right)$, where $f_{1,3}$ are fermions and $f_{2,4}$ antifermions, of momenta $p_{1, \ldots, 4}$. In the rest frame of this process, this is proportional to $(1+\cos \theta)^{2}$, where $\theta$ is the scattering angle between final- and initial-state fermions. The momentum of the final-state fermion, therefore, points preferentially in the direction of the initial-state fermion's momentum, $\cos \theta \rightarrow 1$. For $d \bar{u} \rightarrow W^{-} \rightarrow \ell^{-} \bar{\nu}$ the charged lepton (a fermion) is more likely to move in the direction of the $d$ quark, while for $u \bar{d} \rightarrow W^{+} \rightarrow \ell^{+} \nu$ the charged lepton (an antifermion) is more likely to move backward. The rapidity distribution of charged 
leptons is therefore subject to a tension between the $W$ production asymmetry, which at positive rapidity favours $W^{+}$over $W^{-}$, and the decay asymmetry, which at positive rapidity favours $\ell^{-}$over $\ell^{+}$. The net result is a distribution that changes sign, becoming negative at large lepton rapidity. This is seen explicitly in the right plot of Fig. 22, from the D0 experiment [41], which also shows the great sensitivity of this quantity to different PDF parameterizations, and the potential to improve their determination.

In $p p$ collisions, assuming again the dominance of the first generation of quarks and $f_{q}^{p}(x) \gg$ $f_{\bar{q}}^{p}(x)(q=u, d)$ at large $x$, the $W$ charge asymmetry takes the form: ${ }^{4}$

$$
A(y)=A(-y) \sim \frac{R\left(x_{\max }\right)-r\left(x_{\min }\right)}{R\left(x_{\max }\right)+r\left(x_{\min }\right)},
$$

where $x_{\max (\min )}=\max (\min )\left(x_{1}, x_{2}\right)$ and $f_{\bar{u}}^{p}(x)=r(x) f_{\bar{d}}^{p}(x)$. The extended rapidity coverage of-
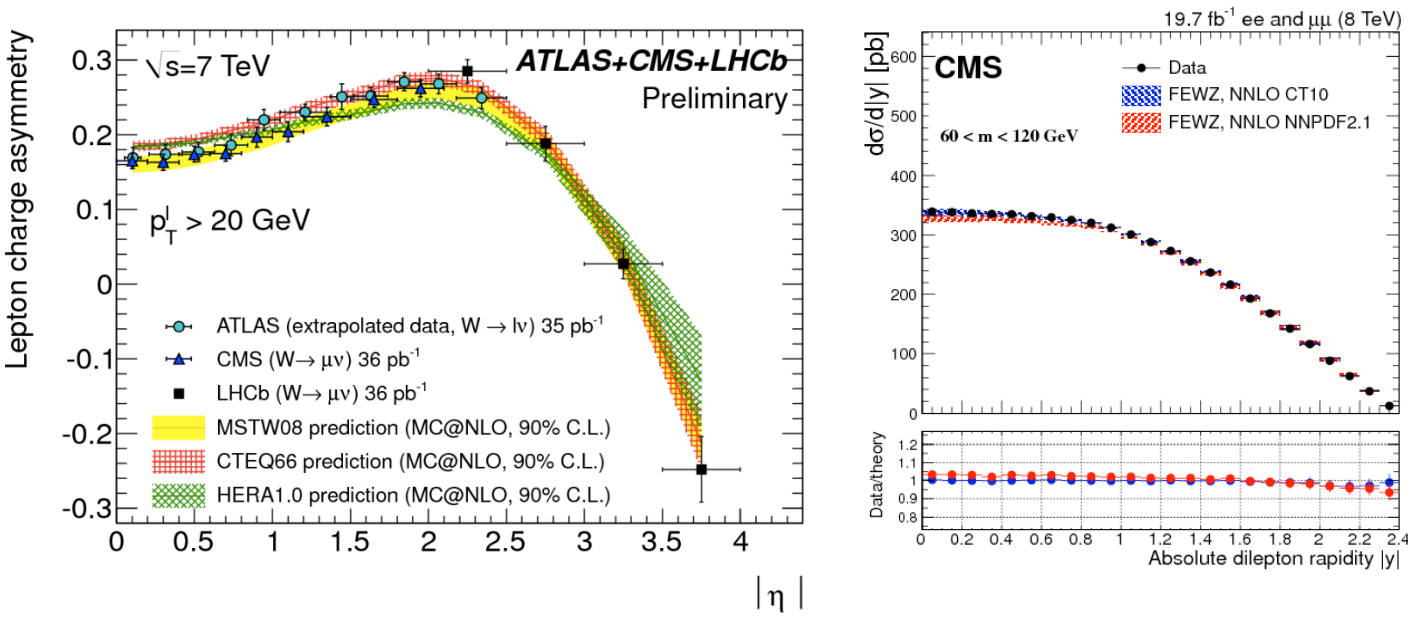

Fig. 23: Left: leptonic charge asymmetries in $W$ production at the LHC $(\sqrt{S}=7 \mathrm{TeV})$, extracted from the measurements of the ATLAS [42], CMS [43] and LHCb [37] experiments. Right: $Z$ boson rapidity spectrum from CMS [44], compared with NNLO predictions [32].

fered by the combination of the ATLAS, CMS and LHCb detectors at the LHC, allows to fully exploit the potential of asymmetry measurements as a probe of the proton structure. This is highlighted in the left plot of Fig. 23, which summarizes the LHC experimental results for the lepton charge asymmetry, obtained at $\sqrt{S}=7 \mathrm{TeV}$, compared to the theoretical predictions based on several sets of PDFs. In particular, notice the large spread of predictions in the largest rapidity regions, spread to be reduced once these data are included as new constraints in global PDF fits (see for example Refs. [45-48]). The PDF sensitivity can be further enhanced by considering the $W$ asymmetry at large rapidity in events produced in association with a high- $p_{T}$ jet, as discussed in Ref. [49].

\subsection{Jet production}

Jet production is the hard process with the largest rate in hadronic collisions. For example, the crosssection for producing jets of transverse energy $E_{T} \gtrsim 100 \mathrm{GeV}$ in $p p$ collisions at the $\operatorname{LHC}\left(\sqrt{S_{\text {had }}}=\right.$ $14 \mathrm{TeV}$ ) is of the order of a $\mu \mathrm{b}$. This means $\sim 10^{4}$ events/s at the luminosities available at the LHC. The data collected during the $8 \mathrm{TeV}$ LHC run extend all the way up to $E_{T} \gtrsim 2 \mathrm{TeV}$, and they will reach

\footnotetext{
${ }^{4}$ It goes without saying that in actual analyses the contributions of all quark and antiquark flavours are taken into account. At the LHC, in particular, the contribution of strange and charm quarks is significant for the $W^{ \pm}$production rate, at the level of $\sim 30 \%$.
} 
$\sim 4-5 \mathrm{TeV}$ by the end of its operations. These events are generated by collisions among partons that carry over $60 \%$ of the available $p p$ energy, and allow one to probe the shortest distances ever reached. The leading mechanisms for jet production are shown in fig. 24.
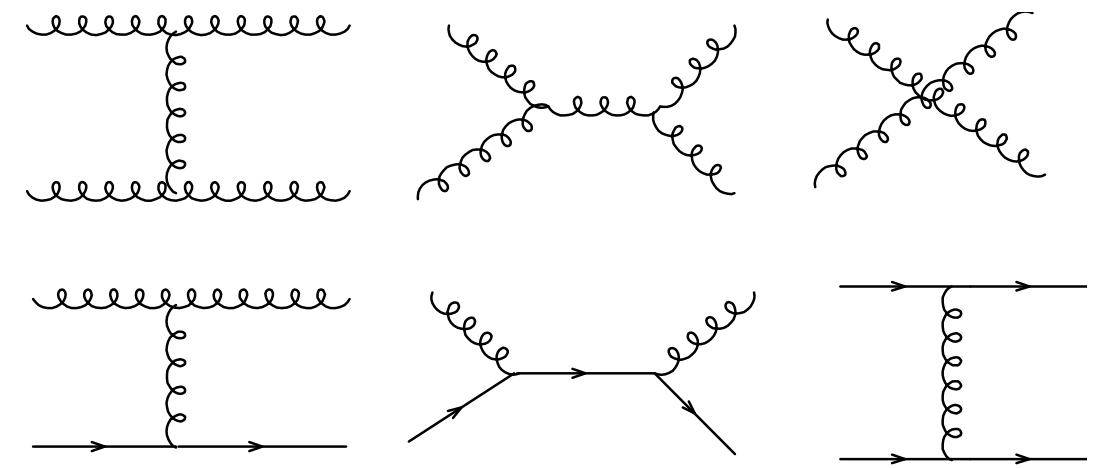

Fig. 24: Representative diagrams for the production of jet pairs in hadronic collisions

The 2-jet inclusive cross-section can be obtained from the formula

$$
d \sigma=\sum_{i j k l} d x_{1} d x_{2} f_{i}^{\left(H_{1}\right)}\left(x_{1}, \mu\right) f_{j}^{\left(H_{2}\right)}\left(x_{2}, \mu\right) \frac{d \hat{\sigma}_{i j \rightarrow k+l}}{d \Phi_{2}} d \Phi_{2}
$$

that has to be expressed in terms of the rapidity and transverse momentum of the quarks (or jets), in order to make contact with physical reality. The two-particle phase space is given by

$$
d \Phi_{2}=\frac{d^{3} k}{2 k^{0}(2 \pi)^{3}} 2 \pi \delta\left(\left(p_{1}+p_{2}-k\right)^{2}\right)
$$

and, in the centre-of-mass of the colliding partons, we get

$$
d \Phi_{2}=\frac{1}{2(2 \pi)^{2}} d^{2} k_{T} d y 2 \delta\left(\hat{s}-4\left(k^{0}\right)^{2}\right)
$$

where $k_{T}$ is the transverse momentum of the final-state partons. Here $y$ is the rapidity of the produced parton in the parton centre-of-mass frame. It is given by

$$
y=\frac{y_{1}-y_{2}}{2}
$$

where $y_{1}$ and $y_{2}$ are the rapidities of the produced partons in the laboratory frame (in fact, in any frame). One also introduces

$$
y_{0}=\frac{y_{1}+y_{2}}{2}=\frac{1}{2} \log \frac{x_{1}}{x_{2}}, \quad \tau=\frac{\hat{s}}{S_{h a d}}=x_{1} x_{2} .
$$

We have

$$
d x_{1} d x_{2}=d y_{0} d \tau
$$

We obtain

$$
d \sigma=\sum_{i j k l} d y_{0} \frac{1}{S_{h a d}} f_{i}^{\left(H_{1}\right)}\left(x_{1}, \mu\right) f_{j}^{\left(H_{2}\right)}\left(x_{2}, \mu\right) \frac{d \hat{\sigma}_{i j \rightarrow k+l}}{d \Phi_{2}} \frac{1}{2(2 \pi)^{2}} 2 d y d^{2} k_{T}
$$

which can also be written as

$$
\frac{d \sigma}{d y_{1} d y_{2} d^{2} k_{T}}=\frac{1}{S_{h a d} 2(2 \pi)^{2}} \sum_{i j k l} f_{i}^{\left(H_{1}\right)}\left(x_{1}, \mu\right) f_{j}^{\left(H_{2}\right)}\left(x_{2}, \mu\right) \frac{d \hat{\sigma}_{i j \rightarrow k+l}}{d \Phi_{2}} .
$$


The variables $x_{1}, x_{2}$ can be obtained from $y_{1}, y_{2}$ and $k_{T}$ from the equations

$$
\begin{aligned}
y_{0} & =\frac{y_{1}+y_{2}}{2} \\
y & =\frac{y_{1}-y_{2}}{2} \\
x_{T} & =\frac{2 k_{T}}{\sqrt{S_{\text {had }}}} \\
x_{1} & =x_{T} e^{y_{0}} \cosh y \\
x_{2} & =x_{T} e^{-y_{0}} \cosh y .
\end{aligned}
$$

For the partonic variables, we need $\hat{s}$ and the scattering angle in the parton centre-of-mass frame $\theta$, since

$$
t=-\frac{\hat{s}}{2}(1-\cos \theta), \quad u=-\frac{\hat{s}}{2}(1+\cos \theta) .
$$

Neglecting the parton masses, you can show that the rapidity can also be written as:

$$
y=-\log \tan \frac{\theta}{2} \equiv \eta
$$

\begin{tabular}{|c|c|}
\hline Process & $\frac{d \hat{\sigma}}{d \Phi_{2}}$ \\
\hline$q q^{\prime} \rightarrow q q^{\prime}$ & $\frac{1}{2 \hat{s}} \frac{4}{9} \frac{\hat{s}^{2}+\hat{u}^{2}}{\hat{t}^{2}}$ \\
\hline$q q \rightarrow q q$ & $\frac{1}{2} \frac{1}{2 \hat{s}}\left[\frac{4}{9}\left(\frac{\hat{s}^{2}+\hat{u}^{2}}{\hat{t}^{2}}+\frac{\hat{s}^{2}+\hat{t}^{2}}{\hat{u}^{2}}\right)-\frac{8}{27} \frac{\hat{s}^{2}}{\hat{u} \hat{t}}\right]$ \\
\hline$q \bar{q} \rightarrow q^{\prime} \bar{q}^{\prime}$ & $\frac{1}{2 \hat{s}} \frac{4}{9} \frac{\hat{t}^{2}+\hat{u}^{2}}{\hat{s}^{2}}$ \\
\hline$q \bar{q} \rightarrow q \bar{q}$ & $\frac{1}{2 \hat{s}}\left[\frac{4}{9}\left(\frac{\hat{s}^{2}+\hat{u}^{2}}{\hat{t}^{2}}+\frac{\hat{t}^{2}+\hat{\hat{u}}^{2}}{\hat{s}^{2}}\right)-\frac{8}{27} \frac{\hat{\hat{u}}^{2}}{\hat{s} \hat{t}}\right]$ \\
\hline$q \bar{q} \rightarrow g g$ & $\frac{1}{2} \frac{1}{2 \hat{s}}\left[\frac{32}{27} \frac{\hat{t}^{2}+\hat{u}^{2}}{\hat{t} \hat{u}}-\frac{8}{3} \frac{\hat{t}^{2}+\hat{u}^{2}}{\hat{s}^{2}}\right]$ \\
\hline$g g \rightarrow q \bar{q}$ & $\frac{1}{2 \hat{s}}\left[\frac{1}{6} \frac{\hat{t}^{2}+\hat{u}^{2}}{\hat{t} \hat{u}}-\frac{3}{8} \frac{\hat{t}^{2}+\hat{u}^{2}}{\hat{s}^{2}}\right]$ \\
\hline$g q \rightarrow g q$ & $\frac{1}{2 \hat{s}}\left[-\frac{4}{9} \frac{\hat{s}^{2}+\hat{u}^{2}}{\hat{s} \hat{u}}+\frac{\hat{u}^{2}+\hat{s}^{2}}{\hat{t}^{2}}\right.$ \\
\hline$g g \rightarrow g g$ & $\frac{1}{2} \frac{1}{2 \hat{s}} \frac{9}{2}\left(3-\frac{\hat{t} \hat{u}}{\hat{s}^{2}}-\frac{\hat{s} \hat{u}}{\hat{t}^{2}}-\frac{\hat{s} \hat{t}}{\hat{u}^{2}}\right)$ \\
\hline
\end{tabular}

with $\eta$ usually being referred to as pseudorapidity.

The leading-order Born cross-sections for parton-parton scattering are reported in Table 2.

Table 2: Cross-sections for light parton scattering. The notation is $p_{1} p_{2} \rightarrow k l, \hat{s}=\left(p_{1}+p_{2}\right)^{2}, \hat{t}=\left(p_{1}-k\right)^{2}$, $\hat{u}=\left(p_{1}-l\right)^{2}$.

It is interesting to note that a good approximation to the exact results can easily be obtained by using the soft-gluon techniques introduced in the third lecture. Based on the fact that even at $90^{\circ}$ $\min (|t|,|u|)$ does not exceed $s / 2$, and that therefore everything else being equal a propagator in the $t$ or $u$ channel contributes to the square of an amplitude 4 times more than a propagator in the $s$ channel, it is reasonable to assume that the amplitudes are dominated by the diagrams with a gluon exchanged in the $t$ (or $u$ ) channel. It is easy to calculate the amplitudes in this limit using the soft-gluon approximation. For example, the amplitude for the exchange of a soft gluon among a $q q^{\prime}$ pair is given by:

$$
\left(\lambda_{i j}^{a}\right)\left(\lambda_{k l}^{a}\right) 2 p_{\mu} \frac{1}{t} 2 p_{\mu}^{\prime}=\lambda_{i j}^{a} \lambda_{k l}^{a} \frac{4 p \cdot p^{\prime}}{t}=\frac{2 s}{t} \lambda_{i j}^{a} \lambda_{k l}^{a} .
$$


The $p_{\mu}$ and $p_{\mu}^{\prime}$ factors represent the coupling of the exchanged gluon to the $q$ and $q^{\prime}$ quark lines, respectively (see Eq. (34). Squaring, and summing and averaging over spins and colours, gives

$$
\sum_{\text {colours,spin }}\left|M_{q q^{\prime}}\right|^{2}=\frac{1}{N^{2}}\left(\frac{N^{2}-1}{4}\right) \frac{4 s^{2}}{t^{2}}=\frac{8}{9} \frac{s^{2}}{t^{2}} .
$$

Since for this process the diagram with a $t$-channel gluon exchange is symmetric for $s \leftrightarrow u$ exchange, and since $u \rightarrow-s$ in the $t \rightarrow 0$ limit, the above result can be rewritten in an explicitly $(s, u)$ symmetric way as

$$
\frac{4}{9} \frac{s^{2}+u^{2}}{t^{2}}
$$

which indeed exactly agrees with the result of the exact calculation, as given in Table 2 . The corrections which appear from $s$ or $u$ gluon exchange when the quark flavours are the same or when we study a $q \bar{q}$ process are small, as can be seen by comparing the above result to the expressions in the table.

As another example we consider the case of $q g \rightarrow q g$ scattering. The amplitude will be exactly the same as in the $q q^{\prime} \rightarrow q q^{\prime}$ case, up to the different colour factors. A simple calculation then gives:

$$
\sum_{\text {colours,spin }}\left|M_{q g}\right|^{2}=\frac{9}{4} \bar{\sum}\left|M_{q q^{\prime}}\right|^{2}=\frac{s^{2}+u^{2}}{t^{2}} .
$$

The exact result is

$$
\frac{u^{2}+s^{2}}{t^{2}}-\frac{4}{9} \frac{u^{2}+s^{2}}{u s}
$$

which even at $90^{\circ}$, the point where the $t$-channel exchange approximation is worse, only differs from this latter by no more than $25 \%$.

As a final example we consider the case of $g g \rightarrow g g$ scattering, which in our approximation gives:

$$
\bar{\sum}\left|M_{g g}\right|^{2}=\frac{9}{2} \frac{s^{2}}{t^{2}}
$$

By $u \leftrightarrow t$ symmetry we should expect the simple improvement:

$$
\bar{\sum}\left|M_{g g}\right|^{2} \sim \frac{9}{2}\left(\frac{s^{2}}{t^{2}}+\frac{s^{2}}{u^{2}}\right)
$$

This only differs by $20 \%$ from the exact result at $90^{\circ}$.

Notice that at small $t$ the following relation holds:

$$
\hat{\sigma}_{g g}: \hat{\sigma}_{q g}: \hat{\sigma}_{q \bar{q}}=\left(\frac{9}{4}\right): 1:\left(\frac{4}{9}\right) .
$$

The 9/4 factors are simply the ratios of the colour factors for the coupling to gluons of a gluon $\left(C_{A}\right)$ and of a quark $\left(T_{F}\right)$, after including the respective colour-average factors $\left(1 /\left(N^{2}-1\right)\right.$ for the gluon, and $1 / N$ for the quark. Using Eq. (80), we can then write:

$$
\begin{aligned}
d \sigma_{h a d r} & =\int d x_{1} d x_{2} \sum_{i, j} f_{i}\left(x_{1}\right) f_{j}\left(x_{2}\right) d \hat{\sigma}_{i j} \\
& =\int d x_{1} d x_{2} F\left(x_{1}\right) F\left(x_{2}\right) d \hat{\sigma}_{g g}(g g \rightarrow \text { jets })
\end{aligned}
$$

where the object

$$
F(x)=f_{g}(x)+\frac{4}{9} \sum_{f}\left[q_{f}(x)+\bar{q}_{f}(x)\right]
$$



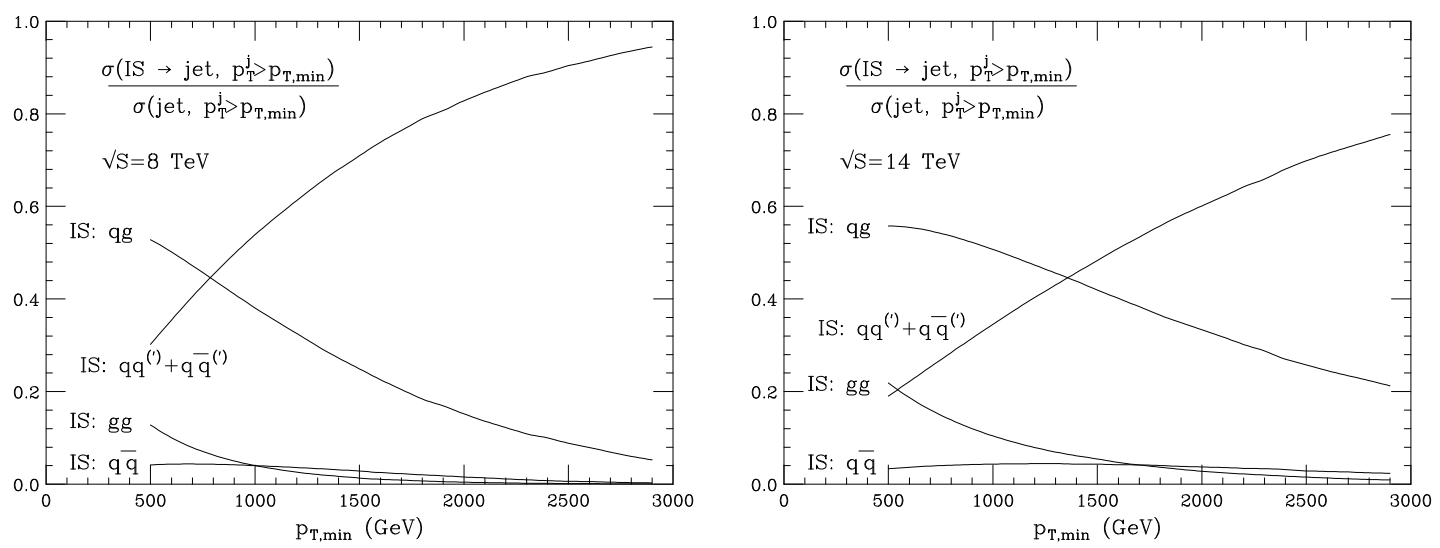

Fig. 25: Relative contribution to the jet- $E_{T}$ rates from the different production channels, in $p p$ collisions at 8 and $14 \mathrm{TeV}$.

is usually called the effective structure function. This result indicates that the measurement of the inclusive jet cross-section does not allow in principle to disentangle the independent contribution of the various partonic components of the proton, unless of course one is considering a kinematical region where the production is dominated by a single process. The relative contributions of the different channels, calculated using current fits of parton densities, are shown for different center of mass energies at the LHC in fig. 25.

\subsection{Jet $E_{T}$ spectra: comparison of theory and experimental data}

Predictions for jet production at colliders are available today at the next-to-leading order in QCD (see the review in Ref. [8]). One of the preferred observables is the inclusive $E_{T}$ spectrum. An accurate

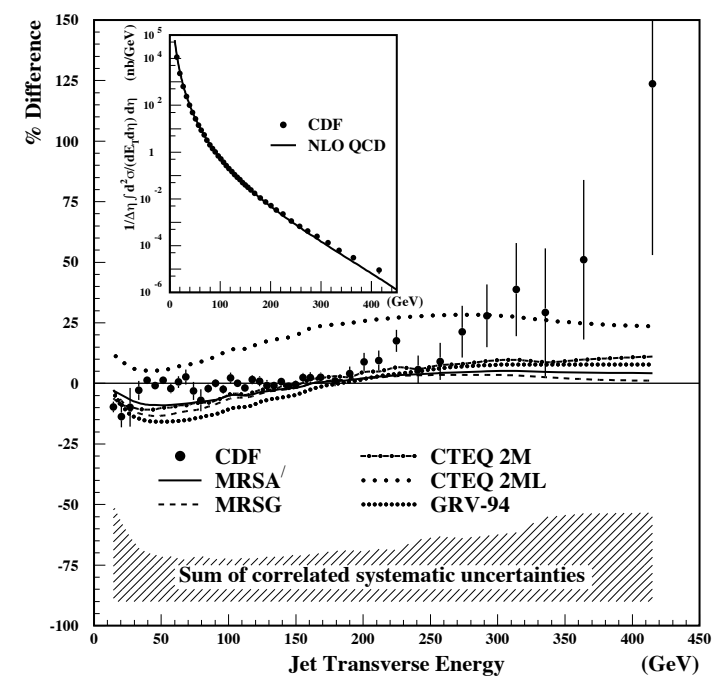

Fig. 26: Comparison of the data vs theory in an early measurement of the jet cross-section at the Tevatron, by the CDF experiment [51]

comparison of data and theory, should it exhibit discrepancies at the largest values of $E_{T}$, could pro- 
vide evidence for new phenomena, such as the existence of a quark substructure. For years it has been known [50] that an underlying quark compositeness would increase the rate of the highest- $E_{T}$ jets. The real question, therefore, is how do we convince ourselves that the prediction is, indeed, accurate. This question became particularly relevant in 1995, when CDF measured a jet cross-section that appeared to deviate from theory in precisely the way predicted by an underlying quark compositeness (see fig. 26). How do we know that this is not due to poorly known quark or gluon densities at large $x$ ? In principle one could incorporate the CDF jet data into a global fit to the partonic PDFs, and verify whether it is possible to modify them so as to maintain agreement with the other data, and at the same time to also fit satisfactorily the jet data themselves. On the other hand, doing this would prevent us from using the jet spectrum as a probe of new physics. In other words, we might be hiding away a possible signal of new physics by ascribing it to the PDFs. Is it possible to have a complementary determination of

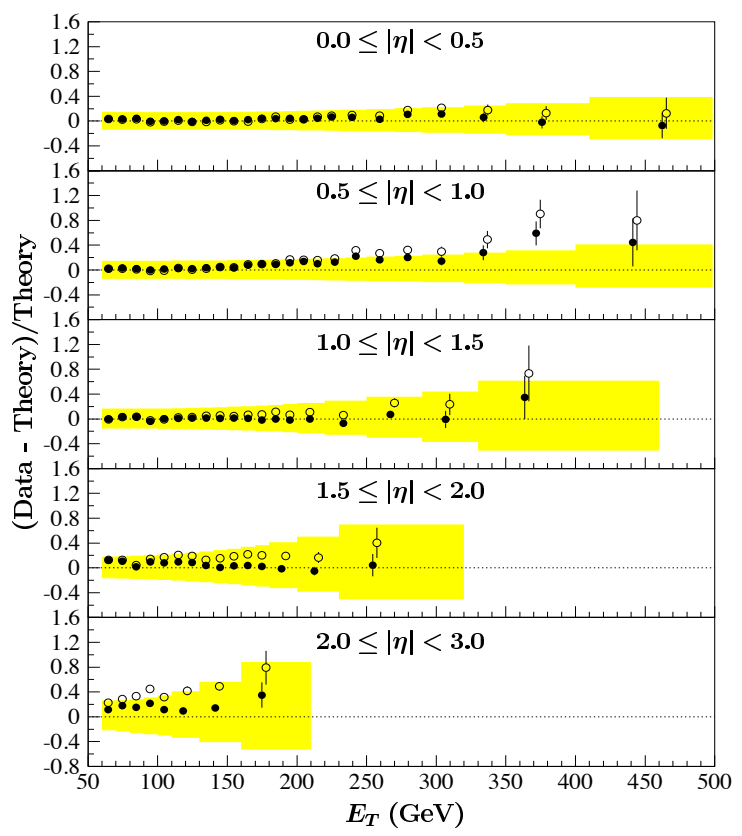

Fig. 27: Inclusive $E_{T}$ spectra for jets in different rapidity regions, as measured at the run 1 of the Tevatron by the D0 Collaboration [52].

the PDF at high- $x$, that could constrain the possible PDF systematics of the jet cross-section and at the same time leave the high- $E_{T}$ tail as an independent and usable observable? This is indeed possible, by fully exploiting the kinematics of dijet production and the wide rapidity coverage of the collider detectors. One could in fact consider final states where the dijet system is highly boosted in the forward or backward region. For example, one could consider cases where $x_{1} \rightarrow 1$ and $x_{2} \ll 1$. In this case, the invariant mass of the dijet system would be small (since $M_{j j}^{2}=x_{1} x_{2} S \ll S$ ), and we know from lower-energy measurements that at this scale jets must behave like pointlike particles, following exactly the QCD-predicted rate. These final states are characterized by having jets at large positive rapidity. One can therefore perform a measurement with forward jets, and use these data to fit the $x_{1} \rightarrow 1$ behaviour of the quark and gluon PDFs without the risk of washing away possible new-physics effects. At that point, the large- $x$ PDFs thus constrained can be safely applied to the kinematical configurations where both $x_{1}$ and $x_{2}$ are large, namely the highest- $E_{T}$ final states, and, if any residual discrepancy between data and theory is observed, infer the possible presence of new physics.

In the case of the Tevatron data, the study of the forward-jet configurations was performed by D0 [52]. Figure 27, from their work, shows the comparison between data and theory for different jetrapidity intervals. Two different PDF sets are used, CTEQ4M, and CTEQ4HJ [53], the latter having 

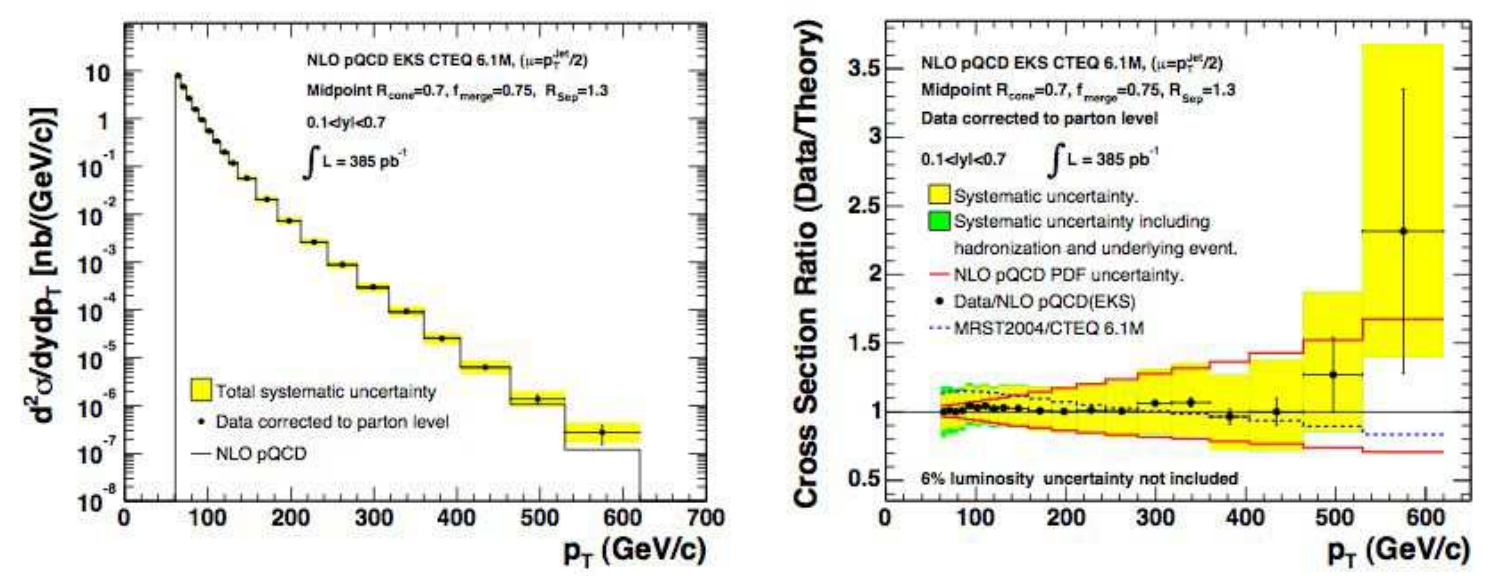

Fig. 28: Inclusive $E_{T}$ spectra for central jets, as measured by the CDF experiment at the Tevatron [54], compared to NLO QCD calculations

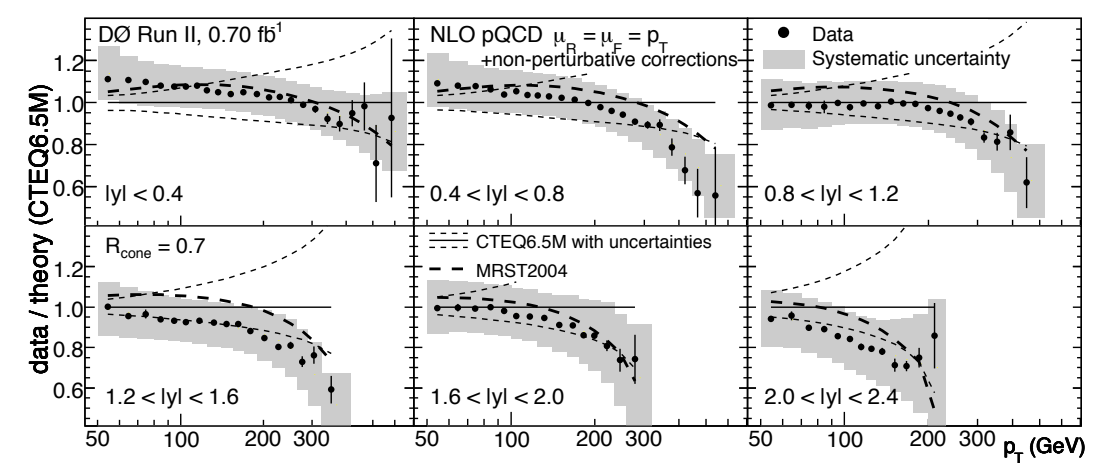

Fig. 29: Comparison of run 2 inclusive jets cross-sections at D0 [55] with QCD calculations. The dashed lines represent the systematics band due to PDF uncertainties

been tuned to describe the CDF high- $E_{T}$ jet tail. Notice the good overall agreement of this prediction for the whole set of rapidities. After this tuning, the residual discrepancy between the CDF high- $E_{T}$ data and QCD is within the theoretical and experimental systematic uncertainties, confirming that jets behave as expected in the Standard Model. This conclusion has been strengthened by the analysis of the run 2 data [54,55], at $\sqrt{S}=1.96 \mathrm{TeV}$, as shown in Figs. 28 and 29 .

\subsection{Jets at the LHC}

The huge statistics and energy lever arm available at the LHC is pushing even further the precision and the sensitivity of jet data. This progress is proceeding hand in hand with the improved theoretical calculations, which have now reached the level of NNLO precision [56].

The experimental systematics, shown in Fig. 30 for the recent final analysis of the ATLAS 8 TeV data [57], are today reduced to less than $10 \%$ for the whole range of jet transverse energies up to $\sim 2 \mathrm{TeV}$, and are dominated by the knowledge of the absolute jet energy scale. The higher statistics available in run 2 of the LHC, and beyond, will allow a further reduction of this systematics, using several experimental handles such as the energy balancing between prompt photons and jets. The theoretical uncertainties include several components. Those of purely perturbative nature are shown in the right plot of Fig. 30, and include the scale, $\alpha_{s}$ and PDF uncertainties. The latter dominate at the largest $E_{T}$ values and, once 

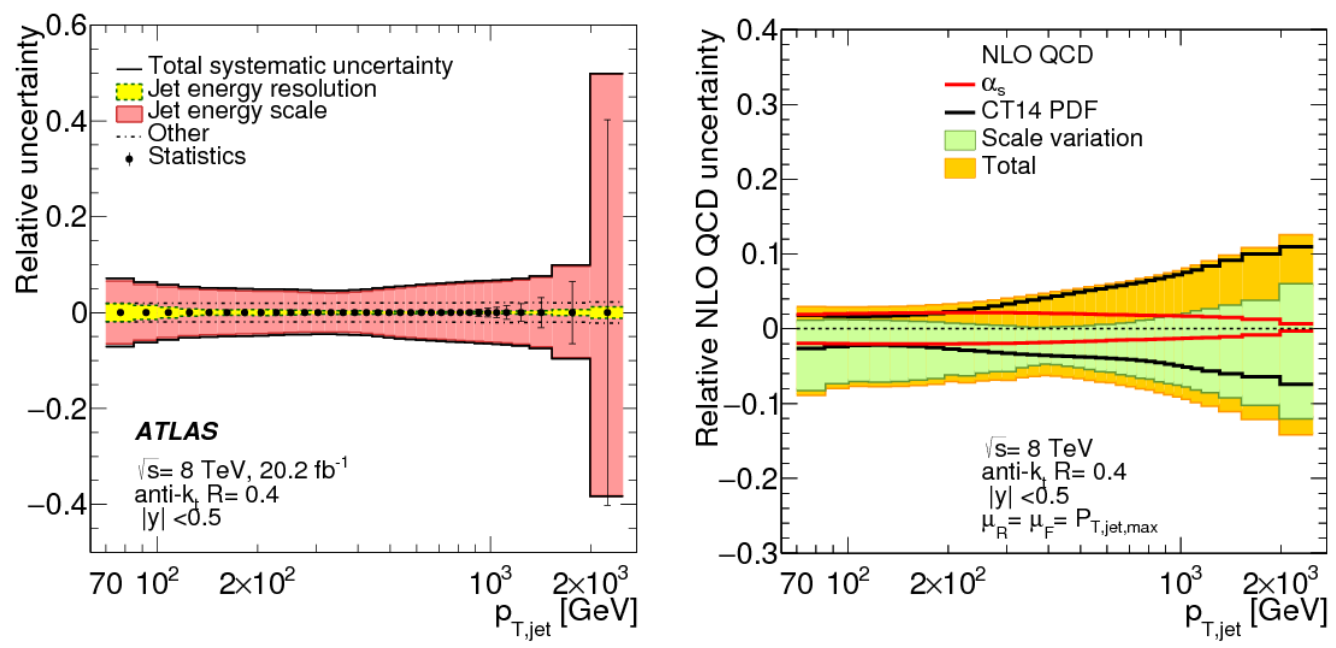

Fig. 30: Experimental (left) and theoretical NLO (right) systematics in the measurement and prediction of the inclusive jet cross section at $\sqrt{S}=8 \mathrm{TeV}$ [57].

again, will likely be reduced with future PDF studies at higher luminosity, along the lines discussed in the previous section in the context of the Tevatron studies.
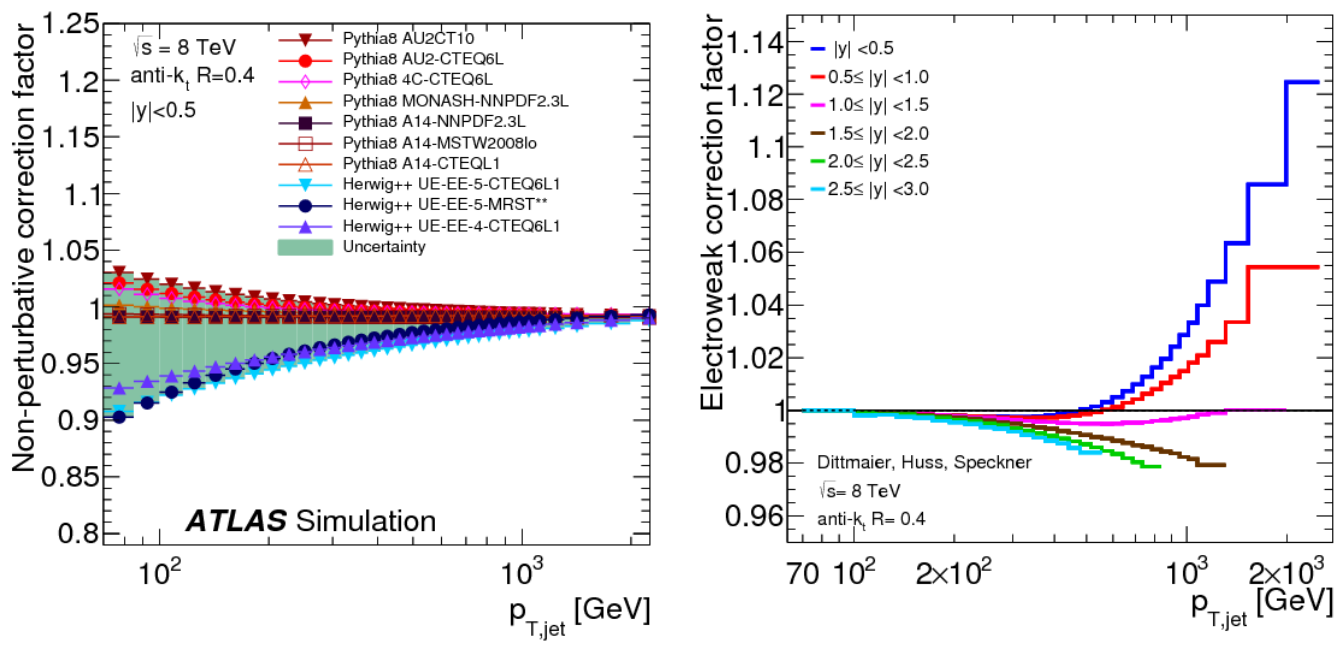

Fig. 31: Hadronization systematics (left) and EW NLO (right) corrections in the theoretical prediction of the inclusive jet cross section at $\sqrt{S}=8 \mathrm{TeV}$ [57].

Further theoretical systematics are related to the non-perturbative corrections needed to translate the jet energy, defined at the perturbative level by the partons, to the actual energy after partons shower and evolve into hadrons. These effects, shown in the left plot of Fig. 31, are enhanced at the lowest energies, and quickly vanish in the interesting multi- $\mathrm{TeV}$ domain. At the highest energies, finally, electroweak (EW) corrections become important, and must be included in the calculations. Their size can reach the $10 \%$ value above the TeV, as shown in the right plot of Fig. 31.

A comparison of data and the NLO theory (corrected for hadronization effects) is shown for the ATLAS measurement [57] in the left plot of Fig. 32. The data extend to $E_{T}$ above $2 \mathrm{TeV}$, covering 10 

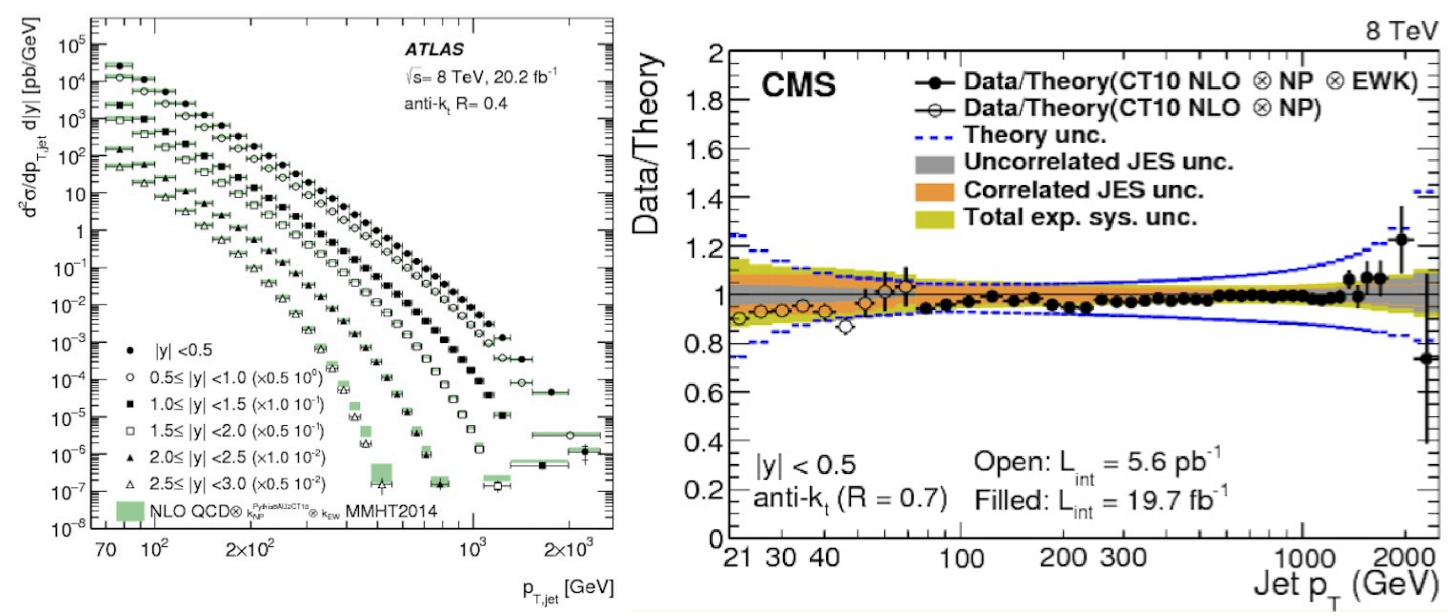

Fig. 32: ATLAS jet cross section data at $\sqrt{S}=8 \mathrm{TeV}$ [57], compared to theoretical calculations (left). Ratio of data and theory for the $8 \mathrm{TeV}$ measurement by CMS [58].

orders of magnitude in rate. The level of agreement is highlighted in the right plot, using the CMS results of the $8 \mathrm{TeV}$ data sample [58]. The agreement is well within the $\pm 10 \%$ overall systematics.

\section{Acknowledgements}

It is a pleasure to thank the organizers of this School for the successful efforts made to bring together top-quality students, in a fantastic environment. 


\section{QCD AND THE PHYSICS OF HADRONIC COLLISIONS}

\section{References}

[1] D.J. Gross, hep-th/9809060.

[2] G. 't Hooft, hep-th/9808154.

[3] R.P. Feynman, Photon-Hadron Interactions (W.A. Benjamin, New York, 1972).

[4] T. Muta, Foundations of QCD (World Scientific, Singapore, 1998).

[5] M.E. Peskin and D.V. Schroeder, An Introduction to Quantum Field Theory (Addison-Wesley, Reading, MA, 1995).

[6] V. Barger and R.J.N. Phillips, Collider Physics (Addison-Wesley, Redwood City, CA, 1997).

[7] R.K. Ellis, W.J. Stirling and B.R. Webber, QCD and Collider Physics (Cambridge University Press, 1996).

[8] J. M. Campbell, J. W. Huston and W. J. Stirling, Rep. Prog. Phys. 70 (2007) 89 [arXiv:hep$\mathrm{ph} / 0611148]$.

[9] J. Butterworth et al., J. Phys. G 43 (2016) 023001 doi:10.1088/0954-3899/43/2/023001 [arXiv:1510.03865 [hep-ph]].

[10] V. Bertone, S. Carrazza and J. Rojo, Comput. Phys. Commun. 185 (2014) 1647 doi:10.1016/j.cpc.2014.03.007 [arXiv:1310.1394 [hep-ph]].

[11] S. Carrazza, A. Ferrara, D. Palazzo and J. Rojo, J. Phys. G 42 (2015) no.5, 057001 doi:10.1088/0954-3899/42/5/057001 [arXiv:1410.5456 [hep-ph]].

[12] R. Field, Applications of Perturbative QCD (Addison-Wesley, Redwood City, CA, 1989).

[13] Yu.L. Dokshitzer, V.A. Khoze, A.H. Mueller and S.I. Troyan, Basics of Perturbative QCD (Editions Frontières, Gif-sur-Yvette, 1991).

[14] V. N. Gribov and L. N. Lipatov, Sov. J. Nucl. Phys. 15 (1972) 438 [Yad. Fiz. 15 (1972) 781].

[15] G. Altarelli and G. Parisi, Nucl. Phys. B 126 (1977) 298.

[16] Y. L. Dokshitzer, Sov. Phys. JETP 46 (1977) 641 [Zh. Eksp. Teor. Fiz. 73 (1977) 1216].

[17] B. R. Webber, Nucl. Phys. B 238 (1984) 492.

[18] B. Andersson, G. Gustafson, G. Ingelman and T. Sjostrand, Phys. Rep. 97 (1983) 31.

[19] S. Gieseke, A. Ribon, M. H. Seymour, P. Stephens and B. Webber, JHEP 0402 (2004) 005 [arXiv:hep-ph/0311208].

[20] R. Hamberg, W. L. van Neerven and T. Matsuura, Nucl. Phys. B 359 (1991) 343 [Nucl. Phys. B 644 (2002) 403].

[21] R. V. Harlander and W. B. Kilgore, Phys. Rev. Lett. 88 (2002) 201801 [hep-ph/0201206].

[22] C. Anastasiou, L. J. Dixon, K. Melnikov and F. Petriello, Phys. Rev. D 69 (2004) 094008 [hep$\mathrm{ph} / 0312266]$.

[23] K. Melnikov and F. Petriello, Phys. Rev. D 74 (2006) 114017 [hep-ph/0609070].

[24] S. Catani, L. Cieri, G. Ferrera, D. de Florian and M. Grazzini, Phys. Rev. Lett. 103 (2009) 082001 [arXiv:0903.2120 [hep-ph]].

[25] R. Gavin, Y. Li, F. Petriello and S. Quackenbush, Comput. Phys. Commun. 182 (2011) 2388 [arXiv:1011.3540 [hep-ph]].

[26] A. Karlberg, E. Re and G. Zanderighi, JHEP 1409 (2014) 134 [arXiv:1407.2940 [hep-ph]].

[27] S. Höche, Y. Li and S. Prestel, Phys. Rev. D 91 (2015) 7, 074015 [arXiv:1405.3607 [hep-ph]].

[28] S. Alioli, C. W. Bauer, C. Berggren, F. J. Tackmann and J. R. Walsh, Phys. Rev. D 92 (2015) 9 , 094020 [arXiv: 1508.01475 [hep-ph]].

[29] S. Dittmaier and M. Kramer, Phys. Rev. D 65 (2002) 073007 [hep-ph/0109062].

[30] U. Baur and D. Wackeroth, Phys. Rev. D 70 (2004) 073015 [hep-ph/0405191].

[31] U. Baur, O. Brein, W. Hollik, C. Schappacher and D. Wackeroth, Phys. Rev. D 65 (2002) 033007 
[hep-ph/0108274].

[32] Y. Li and F. Petriello, Phys. Rev. D 86 (2012) 094034 [arXiv:1208.5967 [hep-ph]].

[33] S. Dittmaier, A. Huss and C. Schwinn, arXiv:1511.08016 [hep-ph].

[34] S. Forte and G. Watt, Ann. Rev. Nucl. Part. Sci. 63 (2013) 291 [arXiv:1301.6754 [hep-ph]].

[35] G. Aad et al. [ATLAS Collaboration], Phys. Rev. D 85 (2012) 072004 [arXiv:1109.5141 [hep-ex]].

[36] S. Chatrchyan et al. [CMS Collaboration], JHEP 1110 (2011) 132 [arXiv:1107.4789 [hep-ex]].

[37] R. Aaij et al. [LHCb Collaboration], JHEP 1412 (2014) 079 [arXiv:1408.4354 [hep-ex]].

[38] T. Aaltonen et al. [CDF Collaboration], Phys. Rev. Lett. 102 (2009) 181801 [arXiv:0901.2169 [hep-ex]].

[39] S. Catani, G. Ferrera and M. Grazzini, JHEP 1005 (2010) 006 [arXiv:1002.3115 [hep-ph]].

[40] R. Gavin, Y. Li, F. Petriello and S. Quackenbush, Comput. Phys. Commun. 184 (2013) 208 [arXiv:1201.5896 [hep-ph]].

[41] V. M. Abazov et al. [D0 Collaboration], Phys. Rev. D 91 (2015) 3, 032007 [Phys. Rev. D 91 (2015) 7, 079901] [arXiv:1412.2862 [hep-ex]].

[42] G. Aad et al. [ATLAS Collaboration], Phys. Lett. B 701 (2011) 31 [arXiv:1103.2929 [hep-ex]].

[43] S. Chatrchyan et al. [CMS Collaboration], Phys. Rev. D 90 (2014) 3, 032004 [arXiv:1312.6283 [hep-ex]].

[44] V. Khachatryan et al. [CMS Collaboration], Eur. Phys. J. C 75 (2015) 4, 147 [arXiv:1412.1115 [hep-ex]].

[45] S. Alekhin, J. Bluemlein and S. Moch, Phys. Rev. D 89 (2014) 5, 054028 [arXiv:1310.3059 [hep$\mathrm{ph}]]$.

[46] R. D. Ball et al. [NNPDF Collaboration], JHEP 1504 (2015) 040 [arXiv:1410.8849 [hep-ph]].

[47] L. A. Harland-Lang, A. D. Martin, P. Motylinski and R. S. Thorne, Eur. Phys. J. C 75 (2015) 5, 204 [arXiv:1412.3989 [hep-ph]].

[48] S. Dulat et al., arXiv:1506.07443 [hep-ph].

[49] S. Farry and R. Gauld, arXiv:1505.01399 [hep-ph].

[50] E. Eichten, K. D. Lane and M. E. Peskin, Phys. Rev. Lett. 50 (1983) 811.

[51] F. Abe et al. [CDF Collaboration], Phys. Rev. Lett. 77 (1996) 438 [arXiv:hep-ex/9601008].

[52] B. Abbott et al. [D0 Collaboration], Phys. Rev. Lett. 86 (2001) 1707 [arXiv:hep-ex/0011036].

[53] H. L. Lai et al., Phys. Rev. D 55 (1997) 1280 [arXiv:hep-ph/9606399].

[54] A. Abulencia et al. [CDF Collaboration], Phys. Rev. D 74 (2006) 071103 [arXiv:hep-ex/0512020].

[55] V. M. Abazov et al. [D0 Collaboration], Phys. Rev. Lett. 101 (2008) 062001 [arXiv:0802.2400 [hep-ex]].

[56] J. Currie, A. Gehrmann-De Ridder, T. Gehrmann, E. W. N. Glover, A. Huss and J. Pires, Phys. Rev. Lett. 119 (2017) no.15, 152001 doi:10.1103/PhysRevLett.119.152001 [arXiv:1705.10271 [hep$\mathrm{ph}]]$.

[57] M. Aaboud et al. [ATLAS Collaboration], JHEP 1709 (2017) 020 doi:10.1007/JHEP09(2017)020 [arXiv:1706.03192 [hep-ex]].

[58] V. Khachatryan et al. [CMS Collaboration], JHEP 1703 (2017) 156 doi:10.1007/JHEP03(2017)156 [arXiv:1609.05331 [hep-ex]]. 